UCSD/PTH 97-14

hep-th/9710024

\title{
Anomaly Matching Conditions and the Moduli Space of Supersymmetric Gauge Theories
}

\author{
Gustavo Dotti and Aneesh V. Manohar \\ Department of Physics, University of California at San Diego, \\ 9500 Gilman Drive, La Jolla, CA 92093-0319
}

(October 1997)

\begin{abstract}
The structure of the moduli space of $N=1$ supersymmetric gauge theories is analyzed from an algebraic geometric viewpoint. The connection between the fundamental fields of the ultraviolet theory, and the gauge invariant composite fields of the infrared theory is explained in detail. The results are then used to prove an anomaly matching theorem. The theorem is used to study anomaly matching for supersymmetric QCD, and can explain all the known anomaly matching results for this case.
\end{abstract}




\section{INTRODUCTION}

One important constraint on the moduli space of vacua of supersymmetric gauge theories [1,2] is that the massless fermions in the low energy theory should have the same flavor anomalies as the fundamental fields, i.e. the 't Hooft consistency conditions should be satisfied [3]. These conditions are considered a particularly stringent test on the spectrum of massless fermions, which is usually obtained from symmetry arguments and renormalization group flows. It is found that for some theories the classical moduli space $\mathcal{M}_{\mathrm{cl}}$ or a suitable quantum modified version $\mathcal{M}_{\Lambda}$ of it satisfies 't Hooft consistency conditions at every point. Other theories fail 't Hooft's test at some vacua, and it is believed that in the infrared, these theories correspond to the weak coupling sector of a dual theory with a different gauge group and matter content [2]. In this paper, sufficient conditions on the fundamental chiral field content $\phi^{i}$ for a theory to satisfy the 't Hooft conditions is established. This is usually done by first finding a basic set of gauge invariants $\hat{\phi}^{j}(\phi)$ and the constraints among them, then checking point by point the matching of flavor anomalies between the fundamental fields and the composites at each point on the moduli space. This procedure involves tedious calculations, and does not offer a systematic approach to the problem of determining whether $\mathcal{M}_{\mathrm{cl}}$ (or a suitable quantum modified version of it) gives the right description of the infrared sector or not. Our results establish simple sufficient conditions on the fundamental fields that guarantee 't Hooft consistency conditions will be satisfied. The novelty of our approach is that no explicit calculation of anomalies is required at any time; it is not even required to know what the basic gauge invariants are.

This paper fills in the details omitted in Ref. «. The outline of the paper is as follows. We devote Sec. II to a review of the connection between $\mathcal{M}_{\mathrm{cl}}$ and the algebraic quotient of $U$ under the action of the complexification $G$ of the gauge group. The results in [5] are rederived and additional information related to the structure of gauge orbits in $U$ and the geometry of $\mathcal{M}_{\mathrm{cl}}$ is provided. Methods to determine the dimension of $\mathcal{M}_{\mathrm{cl}}$ before finding the invariants and their constraints are presented. A number of examples illustrating how naive expectations fail to be true in special situations is also given in this section. In Sec. III, we analyze anomaly matching at points on the classical moduli space. The proof of the anomaly matching theorem makes use of the connection between $\mathcal{M}_{\mathrm{cl}}$ and the vector space $U$ spanned by the fundamental matter fields $\phi^{i}$ provided by the map $\pi: U \rightarrow \mathcal{M}_{\mathrm{cl}}, \pi(\phi)=\hat{\phi}^{i}(\phi)$. Knowledge of the algebraic geometric construction of $\mathcal{M}_{\mathrm{cl}}$ and details related to this map is essential. In Sec. IV we give a rigorous proof of the fact that the flavor anomalies of the massless modes at the vacuum $\hat{\phi}_{0}$ equal those of the full vector space $V$ of the gauge invariant composites (i.e. ignoring all constraints) when $\mathcal{M}$ is the set of critical points of an invariant superpotential. We apply this result to: (i) extend the matching theorem to cases where the superpotential $W$ is not trivial, (ii) globalize the point-by-point result of the matching theorem, and (iii) prove that anomaly matching conditions are compatible with integrating out fields.

Our results guarantee the matching of flavor anomalies between the UV and $\mathcal{M}_{\mathrm{cl}}$ for the large family of $s$-confining theories introduced in [6] and those theories obtained from them by integrating out matter fields, which have a quantum modified moduli space. As an application, in Sec. $\mathrm{V}$ we analyze in detail the well known case of supersymmetric QCD. We first repeat the analysis of Ref. [1], and find all points at which anomalies match by 
performing explicit calculations, then show how the matching follows readily from our results with virtually no calculations. Extensions of the results to dual theories will be described elsewhere.

\section{SUPERSYMMETRIC VACUA AND ALGEBRAIC GEOMETRY}

In this section, we will review some of the properties of supersymmetric vacua in supersymmetric gauge theories, and their connection with algebraic geometry. Many of the results are well known in the physics or mathematics literature. Including the results here will allow us to define our notation, and also to make the paper more self-contained.

\section{A. SUSY Gauge Theories}

The physical objects that we will consider in this paper are supersymmetric gauge theories with gauge group $G_{r}$, where $G_{r}$ is the direct product of a compact connected semisimple Lie group and (possible) $U(1)$ factors. We will assume that all the $U(1)$ 's are compact, i.e. that the fields have rational charges. The action of the gauge theory is

$$
S[\phi]=\int d^{4} x d^{4} \theta \phi^{\dagger} e^{V} \phi+\int d^{4} x d^{2} \theta\left[\frac{1}{4 g^{2}} \operatorname{Tr} W^{\alpha} W_{\alpha}+W(\phi)+c . c .\right]
$$

where $\phi$ is a set of chiral superfields transforming as a (reducible) representation of $G_{r}$, $W_{\alpha}$ is the gauge chiral superfield, $g$ is the gauge coupling constant, $V$ is the gauge vector superfield,

$$
V=V^{A} T^{A}
$$

$T^{A}$ are the generators of the Lie algebra of $G_{r}$, and $W$ is the superpotential. $V$ and $W_{\alpha}$ are related by $W_{\alpha}=-(1 / 4) \bar{D} \bar{D} D_{\alpha} V$. For simplicity of notation, we have assumed that $G_{r}$ is a simple Lie group with coupling constant $g$; if not, there is a different coupling constant for each simple factor in $G_{r}$. The action Eq. (1) will in general also have a global flavor symmetry group $F$, and a $U(1)_{R}$ symmetry.

The set of inequivalent vacua of a supersymmetric gauge theory is referred to as the moduli space. The classical moduli space $\mathcal{M}_{\mathrm{cl}}$ is determined by studying gauge inequivalent constant field configurations that are critical points of the superpotential $W$, and satisfy the $D$-flatness condition

$$
\phi^{\dagger} T^{A} \phi=0 \quad \forall T^{A} \in \operatorname{Lie}\left(G_{r}\right),
$$

where Lie $\left(G_{r}\right)$ is the Lie algebra of $G_{r}$, and has dimension $d_{G}$. The action Eq. (11) has a larger invariance than the gauge symmetry $G_{r}$. It is also invariant under local transformations of the form

$$
\phi \rightarrow e^{i \Lambda^{A} T^{A}} \phi, \quad \quad e^{C^{A} T_{A}} \rightarrow e^{i\left(\Lambda^{A}\right)^{\dagger} T^{A}} e^{C^{A} T^{A}} e^{-i \Lambda^{A} T^{A}},
$$

with $\Lambda^{A}$ chiral superfields [7]. This implies invariance under $G$, the complexification of $G_{r}$. This result was used in Ref. [5] to show that the moduli space of supersymmetric vacua 
is an algebraic variety. We will review the analysis given in Ref. [5] here because we need additional details about the construction of the moduli space and the structure of $G$-orbits not discussed previously.

Any classical supersymmetric vacuum configuration can have only a constant expectation value for the scalar component of the superfield. Thus classical supersymmetric vacua are a subset of $U$, the vector space of all constant field configurations $\phi$. $U$ has dimension $d_{U}$, the number of chiral superfields. If $\phi$ is a point in $U$, the $G$-orbit of $\phi$ will be denoted by $G \phi$. If $\phi$ is a critical point of the superpotential $W$, then so are all points of $G \phi$ since $W$ is $G$-invariant. The set of critical points of $W$ in $U$ will be denoted by $U^{W}$.

\section{B. Algebraic Geometry}

The mathematical objects that we will consider in this paper are affine algebraic sets (a special case of varieties) over the field of complex numbers.' Let $\mathbb{C}\left[x_{1}, \ldots, x_{n}\right]$ be the ring of polynomials in $n$ complex variables $x_{1}, \ldots, x_{n}$. Let $p_{\alpha}\left(x_{1}, \ldots, x_{n}\right), \alpha=1, \ldots, k$ be a finite set of polynomials in the $n$ variables. Then the algebraic set $V\left(p_{\alpha}\right)$ is the set $\left\{x_{i} \in \mathbb{C} \mid p_{\alpha}(x)=0 \forall \alpha\right\}$. It can be thought of as a curve in $\mathbb{C}^{n}$ given implicitly by the polynomial equations $p_{\alpha}=0$. The ideal $\left(p_{1}, \ldots, p_{k}\right)$ is the ideal generated by the polynomials $p_{\alpha}$, i.e. the set of polynomials of the form $\sum_{\alpha} f_{\alpha} p_{\alpha}$, where $f_{\alpha}$ are arbitrary polynomials in $\mathbb{C}\left[x_{1}, \ldots, x_{n}\right]$. Clearly, any polynomial in $\left(p_{1}, \ldots, p_{k}\right)$ vanishes at all points on $V\left(p_{\alpha}\right)$. The set of polynomials that vanish on an algebraic set $X$ (such as $V\left(p_{\alpha}\right)$ ) will be denoted by $I(X)$, and forms a finitely generated ideal of $\mathbb{C}\left[x_{1}, \ldots, x_{n}\right]$. In general, $I\left(V\left(p_{\alpha}\right)\right) \supseteq\left(p_{1}, \ldots, p_{k}\right)$, but equality need not hold. It is possible to define the algebraic set $X$ as the zero set of polynomials $g_{i}, i=1, \ldots, s$ that generate $I(X)$. In this case, $X=V\left(g_{i}\right)$, and $I(X)=\left(g_{1}, \ldots, g_{s}\right)$. If this is the case we say that the equations $g_{i}\left(x_{1}, \ldots, x_{n}\right)=0$, $i=1, \ldots, s$ correctly define $X$.

An irreducible algebraic set is one that cannot be written as a proper union of two algebraic sets. Any algebraic set can be written as a finite union of irreducible algebraic sets in a unique way. The tangent space $T_{p} X$ at a point $p$ of the algebraic set $X \subseteq \mathbb{C}^{n}$ correctly defined by polynomials $g_{i}(x)$ is the vector subspace $\operatorname{ker}\left(\partial g_{i} / \partial x_{j}\right)_{p}$ of $\mathbb{C}^{n}$. The dimension $d_{X}$ of an irreducible algebraic set $X$ is $d_{X}=\min _{p \in X} \operatorname{dim} T_{p} X$. There are alternative equivalent definitions of $T_{p} X$ and different ways of calculating dimensions of irreducible algebraic sets (see [9,8]). The natural complex valued functions on an algebraic set $X$ are the restrictions to $X$ of polynomials in its ambient vector space. These are called regular functions, and the set of regular functions is the coordinate ring $\mathbb{C}[X]$. Note that two polynomials $f_{1}$ and $f_{2}$ in $\mathbb{C}\left[x_{1}, \ldots, x_{n}\right]$ define the same regular function on $X$ if and only if $f_{1}-f_{2} \in I(X)$. This defines an equivalence relation in $\mathbb{C}\left[x_{1}, \ldots, x_{n}\right]$ and, clearly, $\mathbb{C}[X]=\mathbb{C}\left[x_{1}, \ldots, x_{n}\right] / I(X)$. Regular functions from the algebraic set $X \subseteq \mathbb{C}^{n}$ to the algebraic set $Y \subseteq \mathbb{C}^{m}$ are naturally defined to be those that can be written as the restriction to $X$ of $m$ polynomials in $\mathbb{C}^{n}$. All the geometric properties of $X$ are encoded in $\mathbb{C}[X]$, and $X$ can be constructed from $\mathbb{C}[X]$. This fact plays a key role in the discussion of the next subsection.

\footnotetext{
${ }^{1}$ We will use the notation of Refs. [8,9].
} 
It is natural to use the Zariski topology in studying algebraic geometry. The Zariskiclosed sets of $\mathbb{C}^{n}$ are algebraic sets, by definition. The fact that this correctly defines a topology in $\mathbb{C}^{n}$ is non trivial, and is a consequence of the Hilbert basis theorem, which implies that an infinite intersection of algebraic sets is an algebraic set. In the rest of this paper, we will mainly use the Zariski topology, so open and closed sets will always mean with respect to the Zariski topology. In a few places, we will also need to use the more familiar metric-topology on $\mathbb{C}^{n}$, and we will state this explicitly. The Zariski topology might seem a little strange to readers used to thinking about the more familiar metric topology. In $\mathbb{C}$, for example, the Zariski-closed sets are $\mathbb{C}$, a finite set of points, or the null set. Thus the Zariski-closure of the set of integers in $\mathbb{C}$ is the entire space $\mathbb{C}$. In contrast, the metric-closure of the set of integers is itself. Regular functions $f: X \rightarrow Y$ from the algebraic set $X \subseteq \mathbb{C}^{n}$ into the algebraic set $Y \subseteq \mathbb{C}^{m}$ are continuous in the induced Zariski topology, whereas most other functions which are continuous in the metric topology fail to be Zariski continuous. Thus, the Zariski topology allows us to get the strongest results when dealing with regular function. As an example, a regular function $f: \mathbb{C} \rightarrow \mathbb{C}$ that vanishes on the integers $\mathbb{Z}$, must vanish on the closure $\overline{\mathbb{Z}}=\mathbb{C}$, which is a fancy way of saying that the only complex polynomial with infinite roots is the trivial one.

The natural groups to study in algebraic geometry are linear algebraic groups. Let $M_{n}(\mathbb{C})$ be the space of all $n \times n$ complex matrices. $G L(n, \mathbb{C})$ is the set $\operatorname{det} M \neq 0$ in $M_{n}(\mathbb{C})$. $G \subseteq G L(n, \mathbb{C})$ is a linear algebraic group if it is the intersection of $G L(n, \mathbb{C})$ with an algebraic set in $M_{n}(\mathbb{C})$. The group $S U(n)$ is not an algebraic group, because the constraint that the matrix be unitary is not a polynomial relation; it involves complex conjugation. The complexification of $S U(n)$ is the group $S L(n, \mathbb{C})$, which is an algebraic group since the condition that the determinant be unity is a polynomial relation in the elements of the matrix. The complexification of all compact connected semisimple Lie groups are algebraic groups. The vacuum structure of supersymmetric gauge theories will depend on $G$, the complexification of the gauge group $G_{r}$. $G$ is an algebraic group, but $G_{r}$ need not be.

\section{SUSY QED}

It is instructive at this point to go over the well-known example of SUSY QED, which is a $U(1)$ supersymmetric gauge theory with two chiral superfields $Q$ and $\tilde{Q}$ with charges \pm 1 , respectively. The set of all constant field configurations is $\mathbb{C}^{2}=\{(Q, \tilde{Q})\}$ for SQED. In SQED, the group $G=U(1)^{c}$ is the set of transformations $(Q, \tilde{Q}) \rightarrow(z Q, \tilde{Q} / z)$, where $z \in \mathbb{C}, z \neq 0$, as can be seen from Eq. (四). The $G$ orbits are illustrated in Fig. 11. They are the curves $M=\{(Q, \tilde{Q}) \mid Q \tilde{Q}=M \neq 0\}$, the curve $A=\{(Q, 0) \mid Q \neq 0\}$, the curve $B=\{(0, \tilde{Q}) \mid \tilde{Q} \neq 0\}$, and the origin $O=\{(0,0)\}$. The decomposition of $U$ into $G$ orbits is quite complicated, even for the simple example of SQED. $G$ orbits need not be closed. The orbits $M$ and $O$ are closed, and the orbits $A$ and $B$ are not. The closure of the $G$ orbit of $\phi$ is denoted by $\overline{G \phi}$. A very useful result is that the closure of a $G$ orbit in the Zariski topology is equal to its closure in the metric topology. The closure of the two orbits $A$ and $B$ are the curves $(Q, 0)$, and $(0, \tilde{Q})$, where $Q$ and $\tilde{Q}$ are no longer restricted to be non-zero. The closure $\overline{G \phi}$ of orbits $G \phi$ of an algebraic group are irreducible algebraic sets. The closures of 
$G$-orbits are given by the polynomial equations $Q \tilde{Q}=M$ for $\bar{M}, \tilde{Q}=0$ for $\bar{A}, Q=0$ for $\bar{B}$, and $Q=0, \tilde{Q}=0$ for $\bar{O}$, each of which is an irreducible algebraic set.

\section{Algebraic Quotients and the Moduli Space}

The power of algebraic geometry lies in the interplay between geometric properties of the algebraic set $X$ and algebraic properties of its coordinate ring $\mathbb{C}[X]$. The key properties of the ring $\mathbb{C}[X]$ are: (i) $\mathbb{C}[X]$ has no nilpotents (that is, $f \in \mathbb{C}[X], f^{m}=0$ implies $f=0$ ), and (ii) $\mathbb{C}[X]$ is finitely generated. It turns out ( $\mathbb{8}$, chap. I, sect 2 , Thm 1 ) that these are also sufficient conditions for a given ring $\mathcal{A}$ over $\mathbb{C}$ to be isomorphic to the coordinate ring of some algebraic set $X_{\mathcal{A}}, \mathcal{A} \cong \mathbb{C}\left[X_{\mathcal{A}}\right]$. The construction of $X_{\mathcal{A}}$ is in fact very simple: take a set $t_{1}, \ldots, t_{n}$ of generators of $\mathcal{A}$. In general, $\mathcal{A}$ is not a free algebra; there are polynomial equations among the generators $g_{i}\left(t_{1}, \ldots, t_{n}\right)=0, i=1, \ldots, k$. One shows that $X_{\mathcal{A}}=V\left(g_{i}\right) \subseteq \mathbb{C}^{n}$, the equality being valid only in the case of a free algebra. Now assume $X=U^{W} \subseteq U$, the $G$ invariant algebraic set of critical points of a polynomial $G$ invariant superpotential $W$, and let $d_{U}$ be the dimension of $U$. There is a natural representation of $G$ on $\mathbb{C}\left[U^{W}\right]$, namely $\mathbb{C}\left[U^{W}\right] \ni f \rightarrow g \circ f, g \circ f(x) \equiv f\left(g^{-1} x\right), g \in G$. Under this representation the homogeneous polynomials of degree $d$ form invariant subspaces. For reductive linear algebraic groups $(G$ is reductive if any regular representation is completely reducible), the subring $\mathbb{C}\left[U^{W}\right]^{G}$ of $G$ invariant polynomials is finitely generated, as follows from Thm. 4.1.1 in $[9]$. As $\mathbb{C}\left[U^{W}\right]^{G}$ does not have nilpotent elements, it can be thought of as the coordinate ring of an algebraic set $U^{W} / / G=X_{\mathbb{C}\left[U^{W}\right]^{G}}$. If $p_{1}^{G}, \ldots, p_{n}^{G}$ is a minimal set of generators of $\mathbb{C}\left[U^{W}\right]^{G}$, then $U^{W} / / G \subseteq$ $\mathbb{C}^{n}$ is the algebraic set defined by the constraints between the $p_{i}^{G}$ 's. There is a natural regular $\operatorname{map} \pi: U^{W} \rightarrow U^{W} / / G$ by $U^{W} \ni\left(x_{1}, \ldots, x_{d_{U}}\right) \rightarrow\left(p_{1}^{G}\left(x_{1}, \ldots, x_{d_{U}}\right), \ldots p_{n}^{G}\left(x_{1}, \ldots, x_{d_{U}}\right)\right)$ obtained by evaluation of the generating polynomials. The pair $\left(\pi, U^{W} / / G\right)$ is called algebraic (or categorical) quotient of $U^{W}$ under the action of $G$ in the mathematics literature. The basic property of the quotient is that, by construction, $\pi_{*}: \mathbb{C}\left[U^{W} / / G\right] \rightarrow \mathbb{C}\left[U^{W}\right]^{G}$ is an isomorphism (as algebras over $\mathbb{C}$ ) between the coordinate ring of $U^{W} / / G$ and the ring of invariant polynomials in $U^{W}$. Therefore, given any algebraic variety $Z$ and $G$-invariant regular map $f: U^{W} \rightarrow Z$, there exists a unique regular map $\tilde{f}: U^{W} / / G \rightarrow Z$ such that $f=\tilde{f} \circ \pi$, a property that uniquely determines the quotient. Further details of this construction, including properties one through three quoted without proof below, can be found in problems 11.2.6-1,2 in [9], and also in [10]. Using these, we prove other results required to understand the ultraviolet-infrared global anomaly matching. We assume $G$ is reductive, as is always the case in physically interesting theories. Property P5 establishes the anticipated contact with supersymmetric moduli spaces: points in $U^{W} / / G$ are in one to one correspondence with closed orbits in $U^{W}$. As the latter are in one to one correspondence with physically inequivalent classical supersymmetric vacua, $U^{W} / / G$ is the classical moduli space $\mathcal{M}_{\mathrm{cl}}$ of the theory (1). This picture breaks if $G$ fails to be reductive (subsection IIF). The fiber of $\pi(\phi), \pi^{-1}(\pi(\phi))$ is in general a reducible algebraic set. It can be written as the union $\pi^{-1}(\pi(\phi))=X_{1} \cup \ldots \cup X_{n}$ of $G$-invariant irreducible algebraic sets. (Note that $X_{i}$ are $G$-invariant, but need not be a single $G$-orbit.) Then $X_{1} \cap \ldots \cap X_{n}$ is a closed $G$ invariant subset of $\pi^{-1}(\pi(\phi))$, and contains the unique closed $G$ orbit.

One can now connect the algebraic construction of $U^{W} / / G$ discussed above with the classical moduli space $\mathcal{M}_{\mathrm{cl}}$ of supersymmetric vacua [0]. In Ref. [5] it is proven that every 
$G$ orbit contains a (unique) $D$-flat point if and only if the orbit is closed. Every fiber contains exactly one closed $G$ orbit, and thus exactly one $D$-flat point which is also a critical point of $W$. Thus the gauge-inequivalent field configurations that satisfy the $D$-flatness condition and $\nabla W=0$ are in one-to-one correspondence with fibers $\pi^{-1}(\pi(\phi))$. This means that $U^{W} / / G^{c}$ is isomorphic to $\mathcal{M}_{\mathrm{cl}}$ the classical moduli space of the supersymmetric gauge theory, the result shown in Ref. [5].

In studying anomaly matching conditions, one needs to compute anomalies in the full theory (UV theory) and for the massless modes (IR theory). The UV anomalies are computed using the fields $\phi$, i.e. using $U$, and the IR anomalies are computed using the classical moduli space $\mathcal{M}_{\mathrm{cl}}$ or its quantum modification $\mathcal{M}_{\Lambda}$. The classical moduli space $\mathcal{M}_{\mathrm{cl}} \sim U / / G$, so the anomaly matching conditions require knowledge of the structure of the $G$-orbits in $U$.

\section{E. Collection of Mathematical Results}

We will collect here a number of useful results from Refs. [9,10] that will be needed later in this article. Some of them have already been discussed earlier in this section. The basic setting is the algebraic quotient $\pi: U^{W} \rightarrow U^{W} / / G$ of the variety $U^{W}$ given by the critical points of $W$ under the action of a reductive algebraic group $G$.

(P1) $\pi$ is surjective

(P2) If $C \subset U^{W}$ is closed and $G$-invariant, then $\pi(C)$ is closed in $U^{W} / / G$ and $\left(\left.\pi\right|_{C}, \pi(C)\right)$ is the algebraic quotient of $C$ under the action of $G$.

(P3) If $\left\{Z_{i}\right\}_{i \in I}$ is any collection of closed $G$-invariant subsets of $U^{W}$, then $\pi\left(\cap_{i} Z_{i}\right)=$ $\cap_{i} \pi\left(Z_{i}\right)$.

(P4) If $U^{W}$ is irreducible, $U^{W} / / G$ is irreducible. The proof follows from (P1) and Lemma A.1.16 of Ref. [9].

(P5) There is a unique closed $G$ orbit in every fiber of the map $\pi$.

(P6) Two points $\phi_{i}, i=1,2$ lie in the same fiber if and only if $\overline{G \phi_{1}} \cap \overline{G \phi_{2}} \neq \emptyset$. Đ

From now on, we shall assume that $U^{W}$ is irreducible. Then $\mathcal{M}_{\mathrm{cl}}=U^{W} / / G$ is irreducible, and we can use the results of Chap. 1 Sec. 6.3 of $[8]$.

The dimensions of various objects are defined as follows:

$d_{G}$ : The real dimension of the Lie Algebra Lie $\left(G_{r}\right)$ of the gauge group $G_{r}$, which is equal to the complex dimension of Lie $(G)$.

$d_{U}$ : The dimension of $U$.

\footnotetext{
${ }^{2}$ Fibers are called extended orbits in [5]. This characterization of fibers follows from (P3) and the fact that $\pi(\overline{G \phi})$ consists of a single point.
} 
$d_{U^{W}}$ : The dimension of $U^{W}$.

$d_{V}$ : The number of generators of the ring of $G$-invariant polynomials $\mathbb{C}\left[U^{W}\right]^{G}$.

$d_{\mathcal{M}}$ : The dimension of the classical moduli space $\mathcal{M}_{\mathrm{cl}}=U^{W} / / G \subseteq V=\mathbb{C}^{d_{V}}$.

$d$ : The maximum dimension of a $G$-orbit in $U^{W}$. (Note that $G$-orbits can have different dimensions, so $d$ is the maximum possible dimension. Also, $d \leq d_{G}$.)

$d_{f}$ : The minimum dimension of a fiber in $U^{W}$.

Let $T^{A}, A=1, \ldots, d_{G}$ be a basis for Lie $\left(G_{r}\right)$, the Lie Algebra of the gauge group $G_{r}$. The dimension of a $G$ orbit through $\phi$ equals the rank of the $d_{U} \times d_{G}$ matrix $A(\phi)$ with columns $T^{A} \phi$. Note that the entries of $A(\phi)$ are polynomials of degree one in $\phi$. Let $\mathcal{A}_{j}(\phi)$, $j \leq \min \left(d_{G}, d_{U}\right)$ be the set of all $j \times j$ minors of $A(\phi)$, and $\mathcal{I}_{j}$ the set of polynomials in $\phi$ obtained by taking the determinants of the elements of $\mathcal{A}_{j}$. The rank of $A(\phi)$ equals the maximum value of $j$ for which there is a polynomial in $\mathcal{I}_{j}$ which does not vanish at $\phi$. The set $O_{<j}$ of points in $U$ whose orbits have dimension less than $j$ is the closed set $O_{<j}=V\left(\mathcal{I}_{j}\right)$, obtained by requiring that all $j \times j$ minors have zero determinant. The complement is then an open set. Hence "maximum rank" of a matrix valued function on an algebraic set with regular entries is then an example of an open condition, i.e. it defines an open set. The same type of argument shows that the set of smooth points of an irreducible algebraic set $Y$ is open in $Y$. These results together with a straightforward application of [8] Chap. 1, Sec 6.3 imply

(P7) The following is a list of non-empty open subsets of $U^{W}$ and $\mathcal{M}_{\mathrm{cl}}$. Quantities with a belong to $\mathcal{M}_{\mathrm{cl}}$, and without a ${ }^{\wedge}$ belong to $U^{W}$. The inclusion sign means the left-hand side is a non-empty open set contained in the right-hand side, but equality need not hold.

$$
\begin{aligned}
& \hat{O}_{1} \subseteq\left\{\hat{\phi} \in \mathcal{M}_{\mathrm{cl}} \mid \operatorname{dim}\left(\pi^{-1}(\hat{\phi})\right)=d_{U^{W}}-d_{\mathcal{M}}\right\} . \\
& \hat{O}_{2}=\left\{\hat{\phi} \in \mathcal{M}_{\mathrm{cl}} \mid \hat{\phi} \text { is a regular point of } \mathcal{M}_{\mathrm{cl}}\right\} . \\
& \hat{O}_{3}=\left\{\hat{\phi} \in \mathcal{M}_{\mathrm{cl}} \mid \operatorname{dim}\left(\pi^{-1}(\hat{\phi})\right)=d_{f}\right\} . \\
& O_{i}=\pi^{-1}\left(\hat{O}_{i}\right), i=1,2,3 . \\
& O_{4}=\{\phi \in U \mid \operatorname{dim}(G \phi)=d\} .
\end{aligned}
$$

Using these non-empty open sets, and the result that non-empty open subsets of an algebraic set are dense, and any two non-empty open sets have a non-empty intersection (Lemma A.1.12 in [9]) allows one to prove

(P8) The dimension of $\mathcal{M}_{\mathrm{cl}}$ equals that of $U^{W}$ minus the minimum dimension of a fiber. Proof: take a point $\hat{\phi} \in \hat{O}_{1} \cap \hat{O}_{3}$. Then $d_{U W}-d_{\mathcal{M}}=d_{f}$.

(P9) The minimum dimension of fibers is greater than or equal to the maximum dimension of $G$-orbits. Proof: Take $\phi \in O_{3} \cap O_{4}$. As $\pi$ is $G$-invariant, $T_{\phi} G \phi \subseteq T_{\phi} \pi^{-1}(\pi(\phi))$, so that $d \leq d_{f}$. (Here $T_{p} X$ denotes the tangent space of $X$ at the point $p$.) 
We finally quote an important theorem due to Knop [11], and prove a result that is used in Sec. V

Theorem 1 [Knop] (Ref. [1]) If $G$ is semisimple, $G \phi$ has maximum dimension, and $\pi(\phi)$ is smooth, then $\pi_{\phi}^{\prime}$ is surjective.

Theorem 2 If $\operatorname{dim}\left(G \phi_{0}\right)=d$ (maximal) and $G \phi_{0}$ is closed, then

(a) $\pi^{-1}\left(\pi\left(\phi_{0}\right)\right)=G \phi_{0}$.

(b) $d_{\mathcal{M}}=d_{U^{W}}-d$.

If also $d=d_{G}$ then

(c) $\pi_{\phi_{0}}^{\prime}$ is surjective.

Finally, if $\phi_{0}$ is also a smooth point

(d) $\operatorname{ker} \pi_{\phi_{0}}^{\prime}=\operatorname{Lie}(G) \phi_{0}$ and $\pi\left(\phi_{0}\right)$ is smooth.

Proof: Assume (a) is false, and pick any point $\phi_{1} \in \pi^{-1}\left(\pi\left(\phi_{0}\right)\right) \backslash G \phi_{0}$. Because of (P6), $\overline{G \phi_{1}} \cap G \phi_{0} \neq \emptyset$. Therefore there is a sequence $g_{n} \in G$ and a point $g_{0} \in G$ such that $g_{n} \phi_{1} \rightarrow g_{0} \phi_{0}$. Given any point $g \phi_{0} \in G \phi_{0}, g g_{0}^{-1} g_{n} \phi_{1} \rightarrow g \phi_{0}$, so $g \phi_{0} \in \overline{G \phi_{1}}$. Thus $G \phi_{0}$ is a proper subset of $\overline{G \phi_{1}}$, which implies that $\operatorname{dim}\left(G \phi_{0}\right)<\operatorname{dim}\left(G \phi_{1}\right)$ (Lemma A.1.18 of [9]), i.e. $\operatorname{dim}\left(G \phi_{1}\right)>d$ which is a contradiction, since $G$-orbits have maximum possible dimension $d$.

(b) (P9) and (a) implies $d_{f}=d$, and (b) follows using (P8).

(c) By Luna's theorem [12] $G \phi_{0}$ closed implies there is an étale slice at $\phi_{0}$. In the case $\operatorname{dim} G \phi_{0}=d_{G}$ the existence of the slice implies that any curve $\hat{\phi}(t)$ through $\hat{\phi}_{0}=\hat{\phi}(0)$ can be lifted to a curve $\phi(t)$ in $U^{W}$ satisfying $\phi(0)=\phi_{0}$ and $\pi(\phi(t))=\hat{\phi}(t)$. This is equivalent to the surjectivity of $\pi_{\phi_{0}}^{\prime}$.

(d) If $\phi_{0}$ is smooth then $\operatorname{dim} T_{\phi_{0}} U^{W}=d_{U^{W}}$. From (b) and (c) we have: $d_{\mathcal{M}} \leq$ $\operatorname{dim} T_{\pi\left(\phi_{0}\right)} \mathcal{M}_{\mathrm{cl}}=\operatorname{rank} \pi_{\phi_{0}}^{\prime}=d_{U^{W}}-\operatorname{dim} \operatorname{ker} \pi_{\phi_{0}}^{\prime} \leq d_{U^{W}}-\operatorname{dim} T_{\phi_{0}} G \phi_{0}=d_{U^{W}}-d=d_{\mathcal{M}}$. Thus, these are all equalities and (d) follows.

Conditions (c) and (d) ensure that the tangent to the moduli space is isomorphic to the massless modes of the theory determined from the ultraviolet Lagrangian at the classical level, i.e. by looking at the massless modes of the quadratic part of the Lagrangian in unitary gauge.

\section{F. Examples}

\section{SUSY QED}

We have already discussed this case. The space $X$ is $\mathbb{C}^{2}$, since there are two complex fields $Q$ and $\tilde{Q}$. The ring $\mathbb{C}[X]$ is the ring of polynomials in two indeterminates, $\mathbb{C}[Q, \tilde{Q}]$. The ring $\mathbb{C}[X]^{G}$ is the set of gauge invariant polynomials $\mathbb{C}[Q \tilde{Q}]$, freely generated by the single polynomial $M=Q \tilde{Q}$. The algebraic set $Y$ defined by $\mathbb{C}[Y]=\mathbb{C}[Q \tilde{Q}]=\mathbb{C}[X]^{G}$ is thus C. The map $\pi: X \rightarrow Y$ takes $(Q, \tilde{Q})$ to the point $M=Q \tilde{Q}$ in $Y$. The fiber of a point $M \neq 0 \in Y$ is the closed orbit $M$ discussed in Sec. II . The fiber of $M=0$ is $A \cup B \cup O$. 
Note that $\mathbb{C}=Y=X / / U(1)^{c}=\mathbb{C}^{2} / / U(1)^{c}$ is a smooth algebraic set, with no singular points. Nevertheless the fiber of $M=0$ is different in structure from that of the points $M \neq 0$. The fibers $\pi^{-1}(M)$ are closed irreducible and smooth one-dimensional algebraic sets for $M \neq 0 . \pi^{-1}(0)$ is the reducible and singular algebraic set $Q \tilde{Q}=0$, and contains three $G$ orbits, $A, B$ and $O$. It can be written as the union of two irreducible components, $X_{1}$ given by $Q=0$ and $X_{2}$ given by $\tilde{Q}=0$. Their intersection, $X_{1} \cap X_{2}$ is the origin $O=(0,0)$, and is the unique closed $G$ orbit in $\pi^{-1}(0)$. The smooth points $Z$ of $\pi^{-1}(0)$ form the open set $A \cup B$. The complement $\pi^{-1}(0) \backslash Z$ is the origin $O$ and is a smooth closed algebraic set that contains the unique closed $G$ orbit. $\pi^{\prime}$ is surjective at all points of $\pi^{-1}(M \neq 0)$ and at all points of the fiber $\pi^{-1}(0)$ except $(0,0)$. Thus it is possible in this case to choose a point in $\pi^{-1}(0)$ where $\pi^{\prime}$ is surjective; however this point is not in the unique closed orbit $(0,0)$ in the fiber.

Now consider adding a superpotential $W=Q \tilde{Q}$ to SQED. The critical points of $W$ are $Q=\tilde{Q}=0$, so that $U^{W}$ is a single point, and is $G$-invariant. Note that in this example, the critical points of $W$ do not form a complete fiber. The ideal $I(V(\nabla W))$ is the set of all polynomials of the form $Q f(Q, \tilde{Q})+\tilde{Q} g(Q, \tilde{Q})$, and $I(V(\nabla W))^{G}$ is the set of all polynomials of the form $Q \tilde{Q} h(Q \tilde{Q})$, where $f, g$ and $h$ are arbitrary polynomials in their arguments. The classical moduli space $\mathcal{M}_{\mathrm{cl}}$ without a superpotential is the complex plane $\mathbb{C}$ given by $M=Q \tilde{Q}$. Including $W$ restricts one to $\mathcal{M}_{\mathrm{cl}}^{W} \subseteq \mathcal{M}_{\mathrm{cl}}$ given by the algebraic set $M=0$ in $\mathcal{M}_{\mathrm{cl}}$. Since $W$ is gauge invariant, one can rewrite $W$ in terms of the gauge invariant polynomials used to describe the moduli space. In the present example, $W=Q \tilde{Q}=M$. However, when regarded as a function of the gauge invariants rather than the fundamental fields, the critical points of the resultant $W$ do not correctly describe the moduli space in the presence of a superpotential. In our example, $W=M$ has no critical points, whereas $\mathcal{M}_{\mathrm{cl}}^{W}$ contains the single point $M=0 \in \mathcal{M}_{\mathrm{cl}}$. One can obtain $\mathcal{M}_{\mathrm{cl}}^{W}$ by minimizing $W=M^{2}$, instead of $W=M$. However, in general, one cannot obtain the equations defining the moduli space $\mathcal{M}_{\mathrm{cl}}^{W}$ by minimizing a superpotential in the space of gauge invariants.

\section{SUSY QED with equal charges}

An interesting example is SUSY QED with fields $Q_{1}$ and $Q_{2}$ both with charge +1 . This is an anomalous theory, but still provides a useful example that illustrates the structure of fibers. Similar results can be found in more complicated anomaly free theories (such as SUSY QCD with $N_{f}=N_{c}$ ). The space $U$ is $\mathbb{C}^{2}$, and $\mathbb{C}[U]=\mathbb{C}\left[Q_{1}, Q_{2}\right]$. There are no gauge invariant polynomials other than constants, so $\mathbb{C}[U]^{G}=\mathbb{C}$, and the moduli space $\mathcal{M}_{\mathrm{cl}}=U / / U(1)^{c}$ is the zero dimensional space consisting of a single point $P$. The orbits of all points other than $(0,0)$ are radial lines (see Fig. 2), and are not closed. The orbit of $(0,0)$ is a single point, and is the unique closed orbit. The fiber $\pi^{-1}(P)$ is the entire plane $\mathbb{C}^{2}$, and is irreducible. In this case $d_{U}=2, d_{V}=0, d_{\mathcal{M}}=0, d_{G}=1, d=1$ and $d_{f}=2$. Note that $d_{f}>d$, so that the minimum dimension of a fiber can be strictly greater than the maximum dimension of an orbit. 


\section{An example where $\pi^{\prime}$ is not surjective for an orbit of maximal dimension}

This example shows that Theorems 1 and 2 are the most we can say about surjectivity of $\pi^{\prime}$ above smooth points of $\mathcal{M}_{\mathrm{cl}}$. Consider a $U(1)$ theory with three fields $Q_{2}, Q_{+}$and $Q_{-}$ with charges 2,1 and -1 (an anomalous theory). The space $U$ is $\mathbb{C}^{3}$. The gauge invariant polynomials are generated by $A=Q_{+} Q_{-}$and $B=Q_{2} Q_{-}^{2}$. The moduli space $\mathcal{M}_{\mathrm{cl}}$ and $V$ are both $\mathbb{C}^{2}$, since there are no relations among $A$ and $B . \mathcal{M}_{\mathrm{cl}}$ is smooth everywhere. Consider the orbit of $\phi=\left(0, Q_{+}, 0\right)$. It is the set of all points $\left(0, z Q_{+}, 0\right)$ with $z \neq 0$, and has dimension one, i.e. equal to the dimension of the gauge group. However,

$$
\operatorname{rank} \pi^{\prime}=\operatorname{rank}\left(\begin{array}{ccc}
0 & Q_{-} & Q_{+} \\
Q_{-}^{2} & 0 & 2 Q_{2} Q_{-}
\end{array}\right) \leq 1\left(\text { if } Q_{-}=0\right)
$$

so $\pi^{\prime}$ is not surjective even though the orbit has maximal dimension and lies above a smooth point. Note that Theorem 1 does not apply here because the gauge group is not semisimple, and Theorem 2 does not apply here, because the orbit is not closed.

\section{SUSY QCD}

This is the example studied in detail in Refs. [1,2]. The gauge group is $G_{r}=S U(N)$, with $N_{F}$ matter fields $Q^{i \alpha}, i=1, \ldots, N_{F}, \alpha=1, \ldots, N$ in the fundamental $N$ representation of $S U(N)$, and $N_{F}$ matter fields $\tilde{Q}_{\beta j}, j=1, \ldots, N_{F}, \beta=1, \ldots, N$ in the $\bar{N}$ representation of $S U(N)$. The ultraviolet space $U$ is a vector space of dimension $d_{U}=2 N N_{F}$. The complexification of $S U(N)$ is $G=S L(N, \mathbb{C})$, under which $Q^{i \alpha}$ and $\tilde{Q}_{j \beta}$ are in the fundamental and its dual representation. 5 A set of generators of $\mathbb{C}[U]^{G}$, the coordinate ring of gauge invariant polynomials are the mesons and baryons,

$$
\begin{aligned}
M_{j}^{i} & =Q^{i \alpha} \tilde{Q}_{\alpha j} \\
B_{k_{1} \cdots k_{\left(N_{F}-N\right)}} & =\frac{1}{N !} Q^{i_{1} \alpha_{1}} Q^{i_{2} \alpha_{2}} \cdots Q^{i_{N} \alpha_{N}} \epsilon_{\alpha_{1} \alpha_{2} \cdots \alpha_{N}} \epsilon_{i_{1} i_{2} \cdots i_{N} k_{1} \cdots k_{\left(N_{F}-N\right)}} \\
\tilde{B}^{l_{1} \cdots l\left(N_{F}-N\right)} & =\frac{1}{N !} \tilde{Q}_{\alpha_{1} j_{1}} \tilde{Q}_{\alpha_{2} j_{2}} \cdots \tilde{Q}_{\alpha_{N} j_{N}} \epsilon^{\alpha_{1} \alpha_{2} \cdots \alpha_{N}} \epsilon^{j_{1} j_{2} \cdots j_{N} l_{1} \cdots l_{\left(N_{F}-N\right)}} .
\end{aligned}
$$

These polynomials span the vector space $V$. The structure of the classical moduli space $\mathcal{M}_{\text {cl }} \subseteq V$ depends crucially on the value of $N_{F}$.

(i) $\mathbf{N}_{\mathbf{F}}<\mathbf{N}$ : If $N_{F}<N, B$ and $\tilde{B}$ are identically zero. $\mathbb{C}[U]^{G}$ is freely generated by $M_{j}^{i}$, $\mathcal{M}_{\mathrm{cl}}=V$ and $d_{\mathcal{M}}=N_{F}^{2}$. This example illustrates a non-trivial case of dimension counting. Consider the point $\phi$ given by

\footnotetext{
${ }^{3}$ Given a representation of a group $H$ in a vector space $V$ by $v^{i} \rightarrow h_{j}^{i} v^{j}$ we define the dual and conjugate representations on $V^{*}$ by $w_{i} \rightarrow w_{j}\left(h^{-1}\right)_{i}^{j}$ and $w_{i} \rightarrow w_{j}\left(h^{\dagger}\right)_{i}^{j}$. They agree only when the representation of $H$ on $V$ is unitary. Note that the dual representation is defined so as to make $w_{i} v^{i}$ invariant, which is not the case for the conjugate of a non-unitary representation.
} 


$$
Q^{i \alpha}=\tilde{Q}_{\alpha i}= \begin{cases}\delta^{i \alpha} & i \leq N \\ 0 & \text { otherwise }\end{cases}
$$

The $S L(N, \mathbb{C})$ orbit of $\phi$ is closed and of maximum dimension, $\operatorname{dim} G \phi=$ $\operatorname{dim} S L(N, \mathbb{C})-\operatorname{dim} S L\left(N-N_{F}, \mathbb{C}\right)=2 N N_{F}-N_{F}^{2}$. From Theorem 2 we obtain $\operatorname{dim} \mathcal{M}_{\mathrm{cl}}=d_{U}-\operatorname{dim} G \phi=N_{F}^{2}$.

(ii) $\mathbf{N}_{\mathbf{F}}=\mathbf{N}$ : In this case $B=\operatorname{det}(Q), \tilde{B}=\operatorname{det}(\tilde{Q})$, and $\mathbb{C}[U]^{G}$ is not a free algebra, as its generators are constrained by the single relation

$$
\operatorname{det} M-B \tilde{B}=0 .
$$

This gives a hypersurface in $V \cong \mathbb{C}^{N^{2}+2}$, and the dimension of the moduli space is $d_{\mathcal{M}}=N^{2}+1$. This number can also be obtained by applying theorem 2 to the closed $G$ orbit of the point $\left(Q^{i \alpha}=\delta^{i \alpha}, \tilde{Q}=0\right)$

(iii) $\mathbf{N}_{\mathbf{F}}=\mathbf{N}+1$ with a superpotential: When $N_{F}=N+1$ the fields (6) are subject to the following algebraic constraints

$$
\operatorname{Cof}(M)_{i}^{j}-\tilde{B}^{j} B_{i}=0, \quad M_{j}^{i} B_{i}=0, \quad M_{j}^{i} \tilde{B}^{j}=0,
$$

where $\operatorname{cof}(M)_{j}^{i}$ is the matrix of cofactors. Assume we add a superpotential $W=$ $m Q^{N+1 \alpha} \tilde{Q}_{\alpha N+1}$, which is a mass term for the $N+1^{\text {th }}$ flavor. The set $U^{W}$ of critical points of $W$ is just the space $U$ for $N_{F}=N$, naturally embedded in $U$ for $N_{F}=N+1$ by setting the components of $Q$ and $\tilde{Q}$ for the $N+1^{\text {th }}$ flavor to zero. $\pi\left(U^{W}\right)$ is the intersection of the moduli space Eq. (8) with the subspace $B_{i}=\tilde{B}^{i}=M_{i}^{N+1}=$ $M_{N+1}^{i}=0, i=1, \ldots N$ This reproduces Eq. (7), as anticipated by P2 above.

\section{An example involving a non-reductive group}

This example is from [10]. The abelian group $G=\mathbb{C}^{+}$of complex numbers under addition (which is the complexification of the group $G_{r}$ of real numbers under addition) is a simple example of a non-reductive group. We will consider the representation on $U=\mathbb{C}^{2}$ given by $(x, y) \rightarrow(x+z y, y), z \in \mathbb{C}^{+}$. Note that $(x, 0)$ is an invariant subspace with no invariant complement. $\mathbb{C}^{+}$is the linear algebraic non-reductive subgroup of $G L(2, \mathbb{C})$ of upper triangular, determinant one matrices of the form

$$
\left(\begin{array}{ll}
1 & z \\
0 & 1
\end{array}\right)
$$

acting on $\mathbb{C}^{2}$ as the restriction of the fundamental representation of $G L(2, \mathbb{C})$. The orbits $G\left(x_{0}, y_{0}\right)$ are closed one dimensional lines $\left(x, y_{0}\right)$ when $y_{0} \neq 0$. For $y=0$ the orbits are points $\left(x_{0}, 0\right)$ for each value of $x_{0}$ (see Fig. 3 ). Every $G$ orbit is closed. The fibers are the horizontal lines $y=y_{0}$. The $x$-axis $y=0$ is a fiber which contains an infinite number of closed orbits. If this example were an acceptable supersymmetric gauge theory, the algebraic quotient $U / / G$ would not equal the classical moduli space $\mathcal{M}_{\mathrm{cl}}$, since the fiber $y=0$ contains infinitely many closed orbits, i.e. infinitely many inequivalent supersymmetric vacua. 


\section{ANOMALY MATCHING BETWEEN THE ULTRAVIOLET AND INFRARED THEORIES}

Theorem 3: Let $\mathcal{M}_{\mathrm{cl}}$ be the classical moduli space of a supersymmetric gauge theory with gauge group $G_{r}$ and flavor symmetry $F$ and superpotential $W$. It is assumed that the gauge theory has no gauge or gravitational anomalies, and the flavor symmetries have no gauge anomalies. Let $\hat{\phi}_{0} \in \mathcal{M}_{\mathrm{cl}}$ be a point in the classical moduli space. Assume there is a point $\phi_{0} \in U^{W}$ in the fiber $\pi^{-1}\left(\pi\left(\phi_{0}\right)\right)$ of $\hat{\phi}_{0}$ such that

(a) $G$ (the complexification of $G_{r}$ ) is completely broken at $\phi_{0}$, so that $\operatorname{Lie}(G) \phi_{0} \cong \operatorname{Lie}(G)$.

(b) $\operatorname{ker} \pi_{\phi_{0}}^{\prime}=\operatorname{Lie}(G) \phi_{0}$ and $\pi_{\phi_{0}}^{\prime}$ is surjective.

If a subgroup $H \subseteq F$ is unbroken at $\hat{\phi}_{0}$, then the 't Hooft consistency conditions for the $H^{3}$ flavor anomalies and the $H$ gravitational anomalies are satisfied.

For the purposes of the proof, it is convenient to write the original flavor symmetry as $F^{\prime} \times R$, where $R$ is the $R$-symmetry, and $F^{\prime}$ now contains only non- $R$ symmetries. We first prove anomaly matching when $H \subseteq F^{\prime}$ and $W=0$, and then prove consistency for anomalies that include the $R$ symmetry. (Note that the unbroken $R$ symmetry might be a linear combination of the original $R$ symmetry and some generator in $F^{\prime}$.) The extension to $W \neq 0$ follows simply from the results of Sec. [V].

Since $H$ is unbroken at $\hat{\phi}_{0}, \hat{\phi}_{0}$ is $H$-invariant

$$
\operatorname{Lie}(H) \hat{\phi}_{0}=0 \text {. }
$$

The map $\pi: U \rightarrow \mathcal{M}_{\mathrm{cl}}$ commutes with the flavor symmetries, so

$$
0=\operatorname{Lie}(H) \hat{\phi}_{0}=\operatorname{Lie}(H)\left(\pi\left(\phi_{0}\right)\right)=\pi_{\phi_{0}}^{\prime}\left(\operatorname{Lie}(H) \phi_{0}\right) .
$$

Thus, by (a) and (b)

$$
\operatorname{Lie}(H) \phi_{0} \subseteq \operatorname{ker} \pi_{\phi_{0}}^{\prime} \cong \operatorname{Lie}(G)
$$

This implies that given any $\mathfrak{h} \in \operatorname{Lie}(H)$, there is a unique $\mathfrak{g}(\mathfrak{h}) \in \operatorname{Lie}(G)$ such that

$$
\mathfrak{h} \phi_{0}=-\mathfrak{g}(\mathfrak{h}) \phi_{0},
$$

where the minus sign is chosen for convenience. It is straightforward to check that the map Lie $(H) \rightarrow$ Lie $(G)$ given by $\mathfrak{h} \rightarrow \mathfrak{g}(\mathfrak{h})$ is a Lie-algebra homomorphism,

$$
\mathfrak{g}\left(\left[\mathfrak{h}_{1}, \mathfrak{h}_{2}\right]\right)=\left[\mathfrak{g}\left(\mathfrak{h}_{1}\right), \mathfrak{g}\left(\mathfrak{h}_{2}\right)\right] \text {. }
$$

This allows us to define a new "star" representation of Lie $(H)$ in $U$

$$
\mathfrak{h}^{*} \equiv \mathfrak{h}+\mathfrak{g}(\mathfrak{h}) \text {. }
$$

Since Lie $(G) \phi_{0} \in \operatorname{ker} \pi_{\phi_{0}}^{\prime}$, the new Lie $(H)$ representation on $T_{\hat{\phi}_{0}} \mathcal{M}_{\mathrm{cl}}$ defined by $\pi_{\phi_{0}}^{\prime} \mathfrak{h}^{*}$ agrees with the original one. Thus the $\mathfrak{h}^{*}$-anomalies computed at $\hat{\phi}_{0} \in \mathcal{M}_{\mathrm{cl}}$ are the same as the $\mathfrak{h}$-anomalies at the same point. 
Lie $(G) \phi_{0}$ is an invariant subspace under $\mathfrak{h}^{*}$, and the restriction of $\mathfrak{h}^{*}$ to Lie $(G) \phi_{0}$ is the adjoint action by $\mathfrak{g}(\mathfrak{h})$. This can be seen by direct computation. Take any element $\mathfrak{g} \phi_{0} \in \operatorname{Lie}(G) \phi_{0}$. Then

$$
\mathfrak{h}^{*} \mathfrak{g} \phi_{0}=\mathfrak{h} \mathfrak{g} \phi_{0}+\mathfrak{g}(\mathfrak{h}) \mathfrak{g} \phi_{0}=\mathfrak{g} \mathfrak{h} \phi_{0}+\mathfrak{g}(\mathfrak{h}) \mathfrak{g} \phi_{0}=[\mathfrak{g}(\mathfrak{h}), \mathfrak{g}] \phi_{0}=A d_{\mathfrak{g}(\mathfrak{h})} \mathfrak{g} \phi_{0},
$$

since the flavor and gauge symmetries commute, and using Eq. (13). The space $U$ can be broken up into the tangent space to the $G$-orbit $T_{\phi_{0}} G \phi_{0}=\operatorname{Lie}(G) \phi_{0} \cong \operatorname{Lie}(G)$ and its invariant complement, $C_{\phi_{0}}$, since $G$ is reductive. By (b), the map $\pi_{\phi_{0}}^{\prime}$ is a bijective linear map from $C_{\phi_{0}}$ to the tangent space $T_{\hat{\phi}_{0}} \mathcal{M}_{\mathrm{cl}}$ of the moduli space $\mathcal{M}_{\mathrm{cl}}$ at $\hat{\phi}_{0}$, and commutes with $\mathfrak{h}^{*}$. Thus the action of $\mathfrak{h}^{*}$ on $C_{\phi_{0}}$ is equivalent to the action of $\mathfrak{h}$ on $T_{\hat{\phi}_{0}} \mathcal{M}_{\mathrm{cl}}$, by the similarity transformation $S$ given by $\pi_{\phi_{0}}^{\prime}$ restricted to $C_{\phi_{0}}$. One can write

$$
\mathfrak{h}^{*}=\mathfrak{h}_{\mathrm{UV}}+\mathfrak{g}(\mathfrak{h})=\left(\begin{array}{cc}
S \mathfrak{h}_{\mathrm{IR}} S^{-1} & 0 \\
0 & A d_{\mathfrak{g}(\mathfrak{h})}
\end{array}\right),
$$

where the second form shows the structure of $\mathfrak{h}^{*}$ on $U=C_{\phi_{0}} \oplus T_{\phi_{0}} G \phi_{0}$. The action of $\mathfrak{h}$ on $U$ has been labeled by the subscript $\mathrm{UV}$, and the action on the moduli space has been labeled by IR.

One can now compare anomalies in the UV and IR theories using the two different forms for $\mathfrak{h}^{*}$. Since the adjoint representation is real, the $\left(\mathfrak{h}^{*}\right)^{3}$ flavor anomaly and $\mathfrak{h}^{*}$ gravitational anomaly are equal to the anomalies in the infrared theory. All that remains is the proof that the $\left(\mathfrak{h}^{*}\right)^{3}$ and $\mathfrak{h}^{*}$ anomalies of $U$ equal the $\mathfrak{h}^{3}$ and $\mathfrak{h}$ anomalies of $U$. Let $\mathfrak{h}^{\mathrm{A}, \mathrm{B}, \mathrm{C}}$ be any three elements of Lie $(H)$. Then

$$
\begin{aligned}
\operatorname{Tr} \mathfrak{h}^{* \mathrm{~A}}\left\{\mathfrak{h}^{* \mathrm{~B}}, \mathfrak{h}^{* \mathrm{C}}\right\}= & \operatorname{Tr} \mathfrak{h}_{\mathrm{UV}}^{\mathrm{A}}\left\{\mathfrak{h}_{\mathrm{UV}}^{\mathrm{B}}, \mathfrak{h}_{\mathrm{UV}}^{\mathrm{C}}\right\} \\
& +\operatorname{Tr} \mathfrak{g}\left(\mathfrak{h}^{\mathrm{A}}\right)\left\{\mathfrak{h}_{\mathrm{UV}}^{\mathrm{B}}, \mathfrak{h}_{\mathrm{UV}}^{\mathrm{C}}\right\}+\text { cyclic } \\
& +\operatorname{Tr} \mathfrak{h}_{\mathrm{UV}}^{\mathrm{A}}\left\{\mathfrak{g}\left(\mathfrak{h}^{\mathrm{B}}\right), \mathfrak{g}\left(\mathfrak{h}^{\mathrm{C}}\right)\right\}+\text { cyclic } \\
& +\operatorname{Tr} \mathfrak{g}\left(\mathfrak{h}^{\mathrm{A}}\right)\left\{\mathfrak{g}\left(\mathfrak{h}^{\mathrm{B}}\right), \mathfrak{g}\left(\mathfrak{h}^{\mathrm{C}}\right)\right\}
\end{aligned}
$$

The last three lines vanish because the original theory had no gauge and gravitational anomalies, and the flavor symmetries have no gauge anomalies. Thus the $\mathfrak{h}^{3}$ and $\left(\mathfrak{h}^{*}\right)^{3}$ anomalies are the same. Similarly the $\mathfrak{h}^{*}$ and $\mathfrak{h}$ anomalies agree since $\mathfrak{g}$ is traceless because there is no gravitational anomaly. Thus 't Hooft's consistency condition for the flavor anomalies is satisfied.

We now prove the matching theorem for anomalies involving the $R$-charge using an argument similar to the one presented above. The $R$-charge acting on $U$ is given by the matrix $\mathfrak{r}$. The $R$-charge is defined acting on chiral superfields, and so is the charge of the scalar component. Anomalies are computed using the fermionic components, so it is convenient to define a new charge $\tilde{\mathfrak{r}}$ which we will call fermionic $R$-charge, defined by

$$
\tilde{\mathfrak{r}}=\mathfrak{r}-1 .
$$

The anomaly can be computed by taking traces over the chiral superfields of $\tilde{\mathfrak{r}}$. The reason for making the distinction between $\mathfrak{r}$ and $\tilde{\mathfrak{r}}$ is that the map $\pi$ from $U$ to $\mathcal{M}_{\mathrm{cl}}$ commutes with $R=\exp \mathfrak{r}$, but does not commute with $\tilde{R}=\exp \tilde{\mathfrak{r}}$. 
Assume that $R$ is unbroken at $\hat{\phi}_{0}=\pi\left(\phi_{0}\right)$. Then by an argument similar to that above, it is possible to define a "star" $R$-charge, $\mathfrak{r}^{*}$,

$$
\mathfrak{r}^{*} \equiv \mathfrak{r}+\mathfrak{g}(\mathfrak{r})
$$

which has the form

$$
\mathfrak{r}^{*}=\mathfrak{r}_{\mathrm{UV}}+\mathfrak{g}(\mathfrak{r})=\left(\begin{array}{cc}
S \mathfrak{r}_{\mathrm{IR}} S^{-1} & 0 \\
0 & A d_{\mathfrak{g}(\mathfrak{r})}
\end{array}\right)
$$

under the decomposition of $U$ into $C_{\phi_{0}} \oplus T_{\phi_{0}} G \phi_{0}$. As in Eq. (17), we have used the subscripts UV and IR to denote the $R$ charges in the ultraviolet and infrared theories. Note that $S$ is the same matrix in Eqs. (17,21), given by $\pi_{\phi_{0}}^{\prime}$ restricted to $C_{\phi_{0}}$. The fermionic $R$-charge is then given by

$$
\tilde{\mathfrak{r}}^{*}=\mathfrak{r}^{*}-1=\tilde{\mathfrak{r}}_{\mathrm{UV}}+\mathfrak{g}(\mathfrak{r})=\left(\begin{array}{cc}
S \tilde{\mathfrak{r}}_{\mathrm{IR}} S^{-1} & 0 \\
0 & A d_{\mathfrak{g}(\mathfrak{r})}-1
\end{array}\right)
$$

where in the last equality we have used the fact that fermion $R$ charge $\tilde{\mathfrak{r}}_{\mathrm{IR}}=\mathfrak{r}_{\mathrm{IR}}-1$ in the infrared theory.

Compute the trace of $\left(\tilde{\mathfrak{r}}^{*}\right)^{3}$ in $U$,

$$
\operatorname{Tr}\left(\tilde{\mathfrak{r}}^{*}\right)^{3}=\operatorname{Tr}\left\{\tilde{\mathfrak{r}}_{\mathrm{UV}}+\mathfrak{g}(\mathfrak{r})\right\}^{3}=\operatorname{Tr}\left\{\tilde{\mathfrak{r}}_{\mathrm{UV}}^{3}+3 \tilde{\mathfrak{r}}_{\mathrm{UV}}^{2} \mathfrak{g}(\mathfrak{r})+3 \tilde{\mathfrak{r}}_{\mathrm{UV}} \mathfrak{g}(\mathfrak{r})^{2}+\mathfrak{g}(\mathfrak{r})^{3}\right\}
$$

The $R$-charge has no gauge anomaly, so $\operatorname{Tr}_{U} \tilde{\mathfrak{r}}_{\mathrm{UV}}\left\{\mathfrak{g}_{\mathrm{A}}, \mathfrak{g}_{\mathrm{B}}\right\}+\operatorname{Tr}_{\operatorname{Lie}(G)}\left\{A d_{\mathfrak{g}_{\mathrm{A}}}, A d_{\mathfrak{g}_{\mathrm{B}}}\right\}=0$, for any $\mathfrak{g}_{\mathrm{A}, \mathrm{B}} \in \operatorname{Lie}(G)$. Here the first term is the matter contribution to the anomaly, and the second term is the gaugino contribution. The absence of gauge anomalies implies that odd powers of $\mathfrak{g}(\mathfrak{r})$ vanish when traced over the matter fields, since there is no gaugino contribution to these anomalies. Thus we find

$$
\operatorname{Tr}_{U}\left(\tilde{\mathfrak{r}}^{*}\right)^{3}=\operatorname{Tr}_{U}\left(\tilde{\mathfrak{r}}_{\mathrm{UV}}\right)^{3}-3 \operatorname{Tr}_{\operatorname{Lie}(G)} A d_{\mathfrak{g}(\mathfrak{r})}^{2} .
$$

The block diagonal form of $\tilde{\mathfrak{r}}^{*}$ Eq. (22), gives

$$
\operatorname{Tr}_{U}\left(\tilde{\mathfrak{r}}^{*}\right)^{3}=\operatorname{Tr}\left(\tilde{\mathfrak{r}}_{\mathrm{IR}}\right)^{3}-\operatorname{Tr}_{\operatorname{Lie}(G)}\left(1+3 A d_{\mathfrak{g}(\mathfrak{r})}^{2}\right) .
$$

The $R^{3}$ anomaly $A_{\mathrm{UV}}\left(R^{3}\right)$ in the UV theory is given by adding the matter and gaugino contributions

$$
A_{\mathrm{UV}}\left(R^{3}\right)=\operatorname{Tr}_{U}\left(\tilde{\mathfrak{r}}_{\mathrm{UV}}\right)^{3}+\operatorname{Tr}_{\operatorname{Lie}(G)} 1^{3}=\operatorname{Tr}_{U}\left(\tilde{\mathfrak{r}}^{*}\right)^{3}+\operatorname{Tr}_{\operatorname{Lie}(G)}\left(1+3 A d_{\mathfrak{g}(\mathfrak{r})}^{2}\right) .
$$

The $R^{3}$ anomaly $A_{\mathrm{IR}}\left(R^{3}\right)$ in the IR theory is given by

$$
A_{\mathrm{IR}}\left(R^{3}\right)=\operatorname{Tr}\left(\tilde{\mathfrak{r}}_{\mathrm{IR}}\right)^{3},
$$

since there are no gauginos in the low energy theory. Combining Eq. (24 27), one sees immediately that the UV and IR anomalies are equal, $A_{\mathrm{UV}}\left(R^{3}\right)=A_{\mathrm{IR}}\left(R^{3}\right)$.

It is straightforward to check that the gravitational $R$ anomaly, and the $H^{2} R$ and $H R^{2}$ anomalies match. One finds from Eqs. (17,21) that 


$$
\operatorname{Tr} \tilde{\mathfrak{r}}^{*}=\operatorname{Tr} \tilde{\mathfrak{r}}_{\mathrm{UV}}=\operatorname{Tr} \tilde{\mathfrak{r}}_{\mathrm{IR}}-\operatorname{Tr} \operatorname{Lie}(G) 1
$$

which is the matching condition for the gravitational $R$ anomaly, when rewritten as $\operatorname{Tr} \tilde{\mathfrak{r}}_{\mathrm{UV}}+$ $\operatorname{Tr}_{\operatorname{Lie}(G)} 1=\tilde{\mathfrak{r}}_{\mathrm{IR}}$. For the $R H^{2}$ anomaly:

$$
\begin{aligned}
\operatorname{Tr} \tilde{\mathfrak{r}}^{*}\left\{\mathfrak{h}^{* \mathrm{~A}}, \mathfrak{h}^{* \mathrm{~B}}\right\}= & \operatorname{Tr} \tilde{\mathfrak{r}}_{\mathrm{UV}}\left\{\mathfrak{h}_{\mathrm{UV}}^{\mathrm{A}}, \mathfrak{h}_{\mathrm{UV}}^{\mathrm{B}}\right\}+\operatorname{Tr} \mathfrak{g}(\mathfrak{r})\left\{\mathfrak{g}\left(\mathfrak{h}^{\mathrm{A}}\right), \mathfrak{h}_{\mathrm{UV}}^{\mathrm{B}}\right\} \\
& +\operatorname{Tr} \mathfrak{g}(\mathfrak{r})\left\{\mathfrak{h}_{\mathrm{UV}}^{\mathrm{A}}, \mathfrak{g}\left(\mathfrak{h}^{\mathrm{B}}\right)\right\}+\operatorname{Tr} \tilde{\mathfrak{r}}_{\mathrm{UV}}\left\{g\left(h^{\mathrm{A}}\right), g\left(h^{\mathrm{B}}\right)\right\} \\
= & \operatorname{Tr} \tilde{\mathfrak{r}}_{\mathrm{UV}}\left\{h_{\mathrm{UV}}^{\mathrm{A}}, h_{\mathrm{UV}}^{\mathrm{B}}\right\}-\operatorname{Tr} \operatorname{Lie}(G)\left\{A d_{\mathfrak{g}\left(\mathfrak{h}^{\mathrm{A}}\right)}, A d_{\left.\mathfrak{g}\left(\mathfrak{h}^{\mathrm{B}}\right)\right\}}\right.
\end{aligned}
$$

where the last line follows using the fact that there are no $H G^{2}$ anomalies and that the $R G^{2}$ anomaly cancels when the matter and gaugino contributions are added. Using the block diagonal forms of $r^{*}$ and $h^{*}$, one has

$$
\operatorname{Tr} \tilde{\mathfrak{r}}^{*}\left\{\mathfrak{h}^{* \mathrm{~A}}, \mathfrak{h}^{* \mathrm{~B}}\right\}=\operatorname{Tr} \tilde{\mathfrak{r}}_{\mathrm{IR}}\left\{\mathfrak{h}_{\mathrm{IR}}^{\mathrm{A}}, \mathfrak{h}_{\mathrm{IR}}^{\mathrm{B}}\right\}-\operatorname{Tr}_{\operatorname{Lie}(G)}\left\{A d_{\mathfrak{g}\left(\mathfrak{h}^{\mathrm{A}}\right)}, A d_{\mathfrak{g}\left(\mathfrak{h}^{\mathrm{B}}\right)}\right\}
$$

which gives the anomaly matching condition for the $R H^{2}$ anomaly when combined with the previous result. Similarly, for the $R^{2} H$ anomaly:

$$
\begin{aligned}
\operatorname{Tr}\left(\tilde{\mathfrak{r}}^{*}\right)^{2} \mathfrak{h}^{*} & =\operatorname{Tr} \tilde{\mathfrak{r}}_{\mathrm{UV}}^{2} \mathfrak{h}_{\mathrm{UV}}+\operatorname{Tr}\{\mathfrak{g}(\mathfrak{r}), \mathfrak{g}(\mathfrak{h})\} \tilde{\mathfrak{r}}_{\mathrm{UV}}+\operatorname{Tr} \mathfrak{g}(\mathfrak{r})^{2} \mathfrak{h}_{\mathrm{UV}} \\
& =\operatorname{Tr} \tilde{\mathfrak{r}}_{\mathrm{UV}}^{2} \mathfrak{h}_{\mathrm{UV}}-\operatorname{Tr}_{\operatorname{Lie}(G)}\left\{A d_{\mathfrak{g}(\mathfrak{r})}, A d_{\mathfrak{g}(\mathfrak{h})}\right\}
\end{aligned}
$$

The block diagonal forms of $\mathfrak{r}^{*}$ and $\mathfrak{h}^{*}$ give

$$
\operatorname{Tr}\left(\tilde{\mathfrak{r}}^{*}\right)^{2} \mathfrak{h}^{*}=\operatorname{Tr} \tilde{\mathfrak{r}}_{\mathrm{IR}}^{2} \mathfrak{h}_{\mathrm{IR}}-\operatorname{Tr}_{\operatorname{Lie}(G)}\left\{A d_{\mathfrak{g}(\mathfrak{r})}, A d_{\mathfrak{g}(\mathfrak{h})}\right\} .
$$

Comparing with the previous equation shows that the $R^{2} H$ anomalies are the same in the UV and IR theories.

\section{THE INFRARED SECTOR}

The results of the previous section allow one to study the matching of anomalies between the ultraviolet and infrared theories at certain points in the classical moduli space. In this section, we derive some results that allow us to relate the anomaly matching conditions at different points on the moduli space. The moduli space is no longer restricted to be the classical moduli space $\mathcal{M}_{\mathrm{cl}}$.

We consider the case where the moduli space $\mathcal{M}$ is an algebraic curve in an ambient vector space $V$ given as the critical points of a superpotential $W$ with $R$-charge two,

$$
\mathcal{M}=\left\{\hat{\phi} \in V \mid W_{i}(\hat{\phi})=0\right\}
$$

where $\hat{\phi}$ denotes a point in $V$, and we will use the notation $W_{i} \equiv \partial W / \partial \hat{\phi}^{i}, W_{i j} \equiv$ $\partial^{2} W / \partial \hat{\phi}^{i} \partial \hat{\phi}^{j}$, etc. The tangent space to $\mathcal{M}$ at $\hat{\phi}_{0}, T_{\hat{\phi}_{0}} \mathcal{M}$, is defined by

$$
T_{\hat{\phi}_{0}} \mathcal{M}=\left\{\hat{v}^{i} \in V \mid W_{i j}(\hat{\phi}) \hat{v}^{j}=0\right\} .
$$


In all the cases we are interested in, $W$ is a polynomial in $\hat{\phi}$ and Eq. (34) correctly describes the algebraic set, so that Eq. (35) agrees with the algebraic geometry notion of the tangent space.

Assume that a subgroup $H$ of the flavor symmetry group $F$ is unbroken at a point $\hat{\phi}_{0} \in \mathcal{M}$. The invariance of the superpotential $W$ under $F$ implies that

$$
W\left(h_{j}^{i} \hat{\phi}_{0}^{j}\right)=W\left(\hat{\phi}_{0}\right)
$$

where $h_{j}^{i}$ is the matrix for the $H$ transformation in the representation $R$ of the fields $\hat{\phi}$. Differentiating this equation twice with respect to $\hat{\phi}$ and evaluating at the $H$-invariant point $\hat{\phi}_{0}$ gives

$$
h_{i}^{k} h_{j}^{l} W_{k l}\left(h_{j}^{i} \hat{\phi}_{0}^{j}\right)=h_{i}^{k} h_{j}^{l} W_{k l}\left(\hat{\phi}_{0}^{j}\right)=W_{i j}\left(\hat{\phi}_{0}\right),
$$

which shows that $W_{i j}\left(\hat{\phi}_{0}\right)$ is a $H$ invariant tensor that transforms as $(\bar{R} \otimes \bar{R})_{S}$ under $H$, where $R$ is the $H$ representation of the low energy fields $\hat{\phi} \in V$. The tangent space to $\mathcal{M}$ at $\hat{\phi}_{0}$ is the null-space of $W_{i j}$. One can write $V=T_{\hat{\phi}_{0}} \mathcal{M}+N_{\hat{\phi}_{0}} \mathcal{M}$ as the direct sum of the tangent space and its orthogonal complement in $V$. Then $W_{i j}$ provides a non-singular invertible map from $N_{\hat{\phi}_{0}} \mathcal{M}$ into its dual, so that $N_{\hat{\phi}_{0}} \mathcal{M}$ transforms as a real representation of $H$. This immediately implies that the $H$ anomalies computed using the flat directions $T_{\hat{\phi}_{0}} \mathcal{M}$ agree with those computed using the entire vector space $V$.

A similar result holds for the anomalies involving the $R$ charge. Let $R_{i}$ be the $R$-charge of $\hat{\phi}_{i}$, so that

$$
\left.W\left(e^{i \alpha R_{i}} \hat{\phi}_{i}\right)=e^{2 i \alpha} W \hat{\phi}_{i}\right)
$$

since $W$ has $R$ charge two. Differentiating twice with respect to $\hat{\phi}$ shows that

$$
e^{i \alpha\left(R_{i}+R_{j}\right)} W_{i j}\left(\hat{\phi}_{0}\right)=e^{2 i \alpha} W_{i j}\left(\hat{\phi}_{0}\right),
$$

which can be written in the suggestive form

$$
e^{i \alpha\left(\left[R_{i}-1\right]+\left[R_{j}-1\right]\right)} W_{i j}\left(\hat{\phi}_{0}\right)=W_{i j}\left(\hat{\phi}_{0}\right) .
$$

$R_{i}-1$ is the $R$ charge of the fermionic component of the chiral superfield. Thus Eq. (40) shows that $N_{\hat{\phi}_{0}} \mathcal{M}$ transforms like a real representation under $\tilde{R}=R-1$, the fermionic $R$ charge. Thus the $R$ anomalies, and $H \times R$ anomalies can be computed at $\hat{\phi}_{0}$ using $V$ instead of $T_{\hat{\phi}_{0}} \mathcal{M}$. The result can be summarized by

Theorem 4: Let $\mathcal{M} \in V$ be a moduli space described by the critical points of a flavor invariant superpotential $W$ of $R$-charge two. Then the anomalies of an unbroken subgroup $H \subseteq F \times R$ at a point $\hat{\phi}_{0} \in \mathcal{M}$ can be computed using the entire space $V$, instead of the tangent space of $\mathcal{M}$. If the anomaly matching conditions between the UV and IR theories for $H$ are satisfied at $\hat{\phi}_{0}$, they are also satisfied at all points of any moduli space $\mathcal{M}^{\prime} \in V$ given by the critical points of any $W^{\prime}$ (including $W^{\prime}=0$ and $W^{\prime}=W$ ), and at which $H$ is unbroken. 
This result tells us that for moduli spaces described by a superpotential, the precise form of the moduli space is irrelevant. The only role of possible quantum deformations is to remove points of higher symmetry from the moduli space.

One interesting application of this result is to prove that anomaly matching conditions are compatible with integrating out heavy fields. Assume that one has a theory with a moduli space $\mathcal{M}_{\Lambda}$ described by a superpotential $W(\hat{\phi}, \Lambda)$. Now perturb the UV theory by adding a tree level mass term $U(\phi)=m_{i j} \phi^{i} \phi^{j}$ to the superpotential. $U(\phi)$ is gauge invariant, and can be written as a polynomial $W_{m}(\hat{\phi})$ of the gauge invariant composites $\hat{\phi}$ of the IR theory. If the UV theory contains no singlets, then $W_{m}(\hat{\phi})$ is linear in the basic gauge invariant composite fields $\hat{\phi}$. From this, it immediately follows that the effective superpotential of the massive theory is given by

$$
W(\hat{\phi}, \Lambda)=W_{0}(\hat{\phi}, \Lambda)+W_{m}(\hat{\phi}),
$$

where $W_{0}$ is the superpotential in the absence of a mass term, since a linear term in the fields is equivalent to a redefinition of the source.

The anomalies in the IR theory for any unbroken subgroup are unaffected by the change in the moduli space due to the addition of the mass term. They are still obtained by tracing over the whole space $V$. In the UV theory, one should trace not over the whole space $U$, but only over the modes that remain massless when $W_{m}$ is turned on. But it is easy to see that the massive modes in the UV theory form a real representation of the unbroken symmetry. The argument is the same as that used in the IR theory, except that $W_{i j}\left(\hat{\phi}_{0}\right)$ is replaced by the (constant) matrix $m_{i j}$. Thus the mass term does not introduce any modifications to the anomaly in the UV or IR theory. Thus one finds that if the 't Hooft conditions are verified for a theory, they are also valid for any theory obtained by integrating out fields by adding a mass term. The same argument is used to extend Theorem 3 to the case where there is a tree level invariant superpotential $W$. Assume $H \subseteq F$ is unbroken at $\hat{\phi}_{0} \in \mathcal{M}_{\mathrm{cl}}$, and $\phi_{0} \in \pi^{-1}\left(\hat{\phi}_{0}\right) \subseteq U^{W}$ satisfies the conditions of theorem 3. Then theorem 4 applied to the $G \times F$ invariant subset $U^{W} \subseteq U$ gives

$$
\mathcal{A}_{H^{*}}\left(T_{\phi_{0}} U^{W}\right)=\mathcal{A}_{H^{*}}(U),
$$

because $\phi_{0}$ is a fixed point of the $H^{*}$ action and $U^{W}$ is the set of critical points of an $H^{*}$ invariant superpotential. (Here $\mathcal{A}_{H^{*}}(Z)$ denotes the $H^{*}$ anomaly computed using the vector space $Z$.) Thus

$$
\mathcal{A}_{H}\left(T_{\hat{\phi}_{0}} \mathcal{M}_{\mathrm{cl}}\right)=\mathcal{A}_{H^{*}}\left(T_{\phi_{0}} U^{W}\right)=\mathcal{A}_{H^{*}}(U)=\mathcal{A}_{H}(U),
$$

where the first and third equalities are shown in the proof of Theorem 3.

We finally show how to use Theorem 4 to globalize the point-by-point result of the matching theorem for theories with a moduli space given by a superpotential. For a generic supersymmetric gauge theory, the representation $\rho$ of the gauge group on $U$ is made up of $N_{F_{i}}$ tensor copies (flavors) of representations $\rho_{i}, i=1, \ldots, r$ and the non-anomalous flavor symmetry group is

$$
F=S U\left(N_{F_{1}}\right) \times S U\left(N_{F_{2}}\right) \times \cdots \times S U\left(N_{F_{s}}\right) \times U(1)_{1} \times U(1)_{2} \times \cdots \times U(1)_{r-1} \times U(1)_{R} .
$$


The most non-trivial anomaly check is to show that the anomalies for the entire flavor group $F$ match at the origin. From this, anomaly matching at all points of the moduli space follows by another application of Theorem 4 . The non-trivial anomalies are $S U\left(N_{F_{i}}\right)^{3}, S U\left(N_{F_{i}}\right)^{2} U(1)_{j}$, and $U(1)_{i} U(1)_{j} U(1)_{k}$, where the $U(1)_{j}$ include the $R$-symmetry. An $S U\left(N_{F_{i}}\right)^{3}$ anomaly is proportional to the $d$-symbol for the $S U\left(N_{F_{i}}\right)$ group. Since the $d$-symbol is non-zero for $S U(3)$, the $S U\left(N_{F_{i}}\right)$ anomaly can be computed by looking at an $S U(3)$ subgroup. This is a standard trick for computing the anomalies of representations of a general Lie group. To show that the $S U\left(N_{F_{i}}\right)^{3}$ anomaly matches, it is sufficient to find a point on the moduli space that leaves an $S U(3)$ subgroup of $S U\left(N_{F_{i}}\right)$ unbroken. The $S U\left(N_{F_{i}}\right)^{3}$ anomaly must then also match at the origin. Similarly, the $S U\left(N_{F_{i}}\right)^{2} U(1)_{j}$ anomaly matching can be proven by considering a point on the moduli space which leaves a $U(1)$ subgroup of $S U\left(N_{F_{i}}\right)$ and $U(1)_{j}^{\prime}$ unbroken. There is a subtlety here: the unbroken $U(1)_{j}^{\prime}$ generator can be a linear combination of the original $U(1)_{j}$ generator and some of the (broken) $S U\left(N_{F_{k}}\right)$ generators. However, having already proven that the $S U\left(N_{F_{i}}\right)^{3}$ anomaly matches, $S U\left(N_{F_{i}}\right)^{2} U(1)_{j}^{\prime}$ anomaly matching implies $S U\left(N_{F_{i}}\right)^{2} U(1)_{j}$ anomaly matching. The $U(1)_{i} U(1)_{j} U(1)_{k}$ anomaly matching is proven by finding a point where three $U(1)^{\prime \prime}$ 's are unbroken, where again $U(1)^{\prime}$ is a linear combination of the original $U(1)$ 's and $S U\left(N_{F_{i}}\right)$ generators. This procedure might seem complicated, but it is not that involved in practice. In the case of supersymmetric QCD, we will show explicitly how one can prove anomaly matching for $N_{F}=N_{c}+1$ by considering just one point on the moduli space (and its charge conjugate partner).

\section{APPLICATIONS}

The construction of the moduli space of supersymmetric gauge theories can be highly non-trivial when there are many gauge invariant combinations of the fundamental fields. The results of the preceding sections help simplify the analysis of the structure of the moduli space, and of anomaly matching between the fundamental and massless composite fields. We treat the following problems:

(A) Determining the flavor isotropy group $F_{\pi\left(\phi_{0}\right)}$ of the vacuum $\pi\left(\phi_{0}\right) \in \mathcal{M}_{\mathrm{cl}}$, i.e, the maximal unbroken subgroup of $F$ at $\pi\left(\phi_{0}\right)$.'

(B) Setting sufficient conditions for the $F_{\pi\left(\phi_{0}\right)}$ anomalies in $U$ to match the corresponding anomalies in $T_{\pi\left(\phi_{0}\right)} \mathcal{M}_{\mathrm{cl}}$

(C) Setting sufficient conditions for anomalies to match at every vacuum of the (possibly quantum deformed) moduli space.

Three different situations can be considered:

(i) The basic gauge invariants of the given theory are not known.

(ii) The basic gauge invariants are known, but not the constraints among them.

\footnotetext{
${ }^{4}$ This problem is not trivial when the basic gauge invariants of the theory are not known.
} 
(iii) Both the invariant and the constraints are known.

In situation (i) theorems 2 and 3 tell us that if $\phi_{0} \in U^{W}$ is smooth, totally breaks the complexification $G$ of the gauge group $G_{r}$, and has a closed orbit $G \phi_{0}$ (equivalently, there is a D-flat point in $\left.G \phi_{0}\right)$, then the anomalies do match between $U$ and $T_{\pi\left(\phi_{0}\right)} \mathcal{M}_{\mathrm{cl}}$,

$$
A_{F_{\hat{\phi}_{0}}}\left(T_{\hat{\phi}_{0}} \mathcal{M}_{\mathrm{cl}}\right)=A_{F_{\hat{\phi}_{0}}}(U)
$$

This solves problem B. To determine the flavor isotropy group $F_{\pi\left(\phi_{0}\right)}$ we note from Theorem 2 in Section II that for $\phi_{0}$ as above $T_{\phi_{0}}\left(\pi^{-1}\left(\pi\left(\phi_{0}\right)\right)\right)=\operatorname{Lie}(G) \phi_{0}$, and $F_{\pi\left(\phi_{0}\right)}$ is isomorphic to the $G \times F$ isotropy group at $\phi_{0}$.

In situation (ii) we can solve A and B in the same way as in situation (i), but we have the alternative of directly determining $F_{\pi\left(\phi_{0}\right)}$, for which only the gauge invariants and not the constraints among them are needed.

In situation (iii) we have an additional way of dealing with problem B, using the fact that the gauge group $G_{r}$ is semisimple. As $\mathcal{M}_{\mathrm{cl}}$ is known we can calculate its dimension. Suppose $\operatorname{dim} \mathcal{M}_{\mathrm{cl}}=\operatorname{dim} U-d_{G}$ and a point $\phi_{0}$ is found such that: $\pi\left(\phi_{0}\right)$ is smooth, $\phi_{0}$ breaks completely $G$. Then the $F_{\pi\left(\phi_{0}\right)}$ anomalies match between $U$ and $T_{\pi\left(\phi_{0}\right)} \mathcal{M}_{\mathrm{cl}}$. This follows from theorems 1, 2 and 3. Note that it is crucial that $\phi_{0}$ break completely the complexification of the gauge group. Consider the example of supersymmetric QCD with $N_{F}=N_{c}-1$. The classical moduli space is the span of the unconstrained fields $M_{j}^{i}$, so $\mathcal{M}_{\mathrm{cl}} \cong \mathbb{C}^{N_{F}^{2}}$ and $\operatorname{dim}$ $\mathcal{M}_{\mathrm{cl}}=\operatorname{dim} U-d_{G}$. The point $\phi_{0}$ of coordinates $\left(\tilde{Q}_{0}\right)_{\alpha i}=0, Q_{0}^{i \alpha}=m \delta^{i \alpha}, \alpha \leq N_{F}, 0$ for $\alpha=N_{c}$, totally breaks $G_{r}=S U\left(N_{c}\right)$, but not $G=S L\left(N_{c}, \mathbb{C}\right) . \mathcal{M}_{\mathrm{cl}}$ is smooth everywhere, in particular $\pi\left(\phi_{0}\right)=0$ is a smooth point. However, anomalies do not match between the $\mathrm{UV}$ and $T_{0} \mathcal{M}_{\mathrm{cl}}=\operatorname{span}\left(M_{j}^{i}\right)$. Now consider the point $\phi_{1}$ with coordinates $\left(\tilde{Q}_{1}\right)_{\alpha i}=Q_{1}^{i \alpha}=$ $m \delta^{i \alpha}, \alpha \leq N_{F}, 0$ for $\alpha=N_{c}$. This point breaks completely the complexification of the gauge group, and it is mapped onto a smooth point. Theorems 1, 2 and 3 predict anomaly matching, which is straightforward from the fact that $F_{\pi\left(\phi_{1}\right)}$ is the diagonal $S U\left(N_{F}\right)$.

Theorem 4 in Section IV gives an answer to problem $\mathrm{C}$. Whenever $\mathcal{M}_{\mathrm{cl}}$ is the set of critical points of an invariant superpotential, the matching of the $F_{\hat{\phi}_{i}}$ anomalies at the vacua $\hat{\phi}_{i}, i=$ $1, \ldots, s$ implies the matching of $F_{\hat{\phi}_{i}}$ anomalies at any vacuum $\hat{\phi}$ where $F_{\hat{\phi}} \supseteq F_{\hat{\phi}_{i}}$. In particular, we can take $\hat{\phi}=0 \in \mathcal{M}_{\mathrm{cl}}$, where the flavor group is not broken at all. The points $\hat{\phi}_{i}$ can be chosen such that the matching of the $F_{\hat{\phi}_{i}}$ anomalies at the origin (where the tangent space is the full ambient vector space) implies the matching of the full flavor group anomalies there. Applying again Theorem 4 we prove that every point of $\mathcal{M}_{\mathrm{cl}}$ will pass 't Hooft's test. This extends to $\mathcal{M}_{\Lambda}$ if $\mathcal{M}_{\Lambda}$ also comes from a flavor invariant superpotential. This idea can be used to prove anomaly matching for the large family of s-confining theories of ref [6], and for those obtained from them by integrating out any number of flavors. The latter may have a quantum modified moduli space, which can be embedded in the same ambient vector space of the s-confining theory, and is described with an invariant superpotential (Section IV).

Below, we extend the explicit anomaly matching calculations in supersymmetric QCD done in [1] to a generic point in moduli space, and show that anomalies match precisely at those

\footnotetext{
${ }^{5}$ Note that in general $F_{\phi_{0}} \subseteq F_{\pi\left(\phi_{0}\right)}$.
} 
points predicted by our theorems. The amount of calculations using both approaches is contrasted.

\section{A. Supersymmetric QCD}

$$
\text { 1. } N_{c}=2
$$

The quarks and antiquarks transform as the $\mathbf{2}$ of $S U(2)$, and so can be treated together as $2 N_{F}$ flavors of doublets. The flavor symmetry is $S U\left(2 N_{F}\right) \times U(1)_{R}$. The basic gauge invariant field is the meson

$$
V^{i j}=\epsilon_{\alpha \beta} Q^{i \alpha} Q^{j \beta}
$$

The transformation properties of the fundamental fields and composites under the flavor

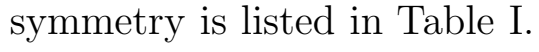

TABLE I. Flavor representation of the fundamental and composite fields for $S U(2)$ gauge theory with $2 N_{F}$ fundamentals.

\begin{tabular}{lcc}
\hline \hline & $S U\left(2 N_{F}\right)$ & $U(1)_{R}$ \\
\hline$Q^{i \alpha}$ & $\square$ & $\frac{N_{F}-2}{N_{F}}$ \\
$V^{i j}$ & $\square$ & $2 \frac{N_{F}-2}{N_{F}}$ \\
\hline \hline
\end{tabular}

The classical moduli space for $N_{F}=1$ (i.e. $N_{F}<N_{C}$ ) is given by all possible values for $V^{i j}$. Since $V^{i j}$ is a $2 \times 2$ antisymmetric matrix, it has the form

$$
V^{i j}=\left(\begin{array}{cc}
0 & v \\
-v & 0
\end{array}\right)
$$

For $v \neq 0$, the unbroken flavor symmetry is flavor $S U(2)$, whereas for $v=0$, the full $S U(2) \times U(1)_{R}$ symmetry is unbroken. It is straightforward to verify that the $S U(2)^{3}$ and $S U(2)$ anomalies match between the UV and IR theories so that the IR and UV theories have the same anomalies at a point on the moduli space where $v \neq 0$. At the origin of moduli space $v=0$, the UV and IR anomalies do not match. The anomaly matching theorem can be used at all points $v \neq 0$. One can pick a point

$$
Q_{0}^{i \alpha}=w\left(\begin{array}{ll}
1 & 0 \\
0 & 1
\end{array}\right)
$$

with $v=w^{2}$, which gives Eq. (46) for $V^{i j}$. The orbit containing $Q_{0}^{i \alpha}$ is closed, and $G$ is completely broken, so the UV and IR anomalies must match. There is no point above the origin $V^{i j}=0$ that satisfies the requirements for applying the anomaly matching theorem. Thus for $N_{F}=1$, all points where the anomalies match can be described using the theorem. Note that the point

$$
Q_{0}^{i \alpha}=w\left(\begin{array}{ll}
1 & 0 \\
0 & 0
\end{array}\right)
$$


completely breaks the gauge group $G_{r}$, but not the complexified gauge group $G$. Completely breaking $G_{r}$ is not sufficient to use theorem 3 .

For $N_{F} \geq 2$, the classical moduli space is the set of all $V^{i j}$ 's subject to the constraint that $\operatorname{rank} V \leq 2[\mathbb{1}$. At a generic point

$$
V^{i j}=\left(\begin{array}{cc|ccc}
0 & v & 0 & \cdots & 0 \\
-v & 0 & 0 & \cdots & 0 \\
\hline 0 & 0 & 0 & \cdots & 0 \\
\vdots & \vdots & \vdots & & \vdots \\
0 & 0 & 0 & \cdots & 0
\end{array}\right)
$$

with $v \neq 0$, the flavor group is broken to $S U(2) \times S U\left(2 N_{F}-2\right) \times U(1)_{R}$. To compute the anomalies in the UV and IR theories, it is convenient to break up the flavor index into $i=1,2$ and $i=3, \ldots 2 N_{F}$. The quarks can be broken into $Q_{1}$ and $Q_{2}$, respectively. The meson $V^{i j}$ can be written as

$$
V^{i j}=\left(\begin{array}{cc}
V_{11} & V_{12} \\
-V_{12}^{T} & V_{22}
\end{array}\right)
$$

where $V_{11}$ and $V_{22}$ are $2 \times 2$ and $\left(2 N_{F}-2\right) \times\left(2 N_{F}-2\right)$ antisymmetric matrices, and $V_{12}$ is a $2 \times 2 N_{F}-2$ matrix. Denoting the tangent vectors to the moduli space by $\delta V$, one sees that the constraint rank $V \leq 2$ requires that $\delta V_{22}=0$ if $v \neq 0$. The flavor transformations of the fields is given in Table 【.

TABLE II. Flavor representation of the fundamental and composite fields for $S U(2)$ gauge theory with $2 N_{F}$ fundamentals under the $S U(2) \times S U\left(2 N_{F}-2\right) \times U(1)_{R}$ subgroup.

\begin{tabular}{lccc}
\hline \hline & $S U(2)$ & $S U\left(2 N_{F}\right)$ & $U(1)_{R}$ \\
\hline$Q_{1}^{i \alpha}$ & $\square$ & - & 0 \\
$Q_{2}^{i \alpha}$ & - & $\square$ & $\frac{N_{F}-2}{N_{F}-1}$ \\
$\delta V_{11}^{i j}$ & - & - & 0 \\
$\delta V_{12}^{i j}$ & $\square$ & $\square$ & $\frac{N_{F}-2}{N_{F}-1}$ \\
\hline \hline
\end{tabular}

It is easy to verify that the flavor anomalies of $Q_{1}$ and $Q_{2}$ are the same as those of $\delta V_{11}$ and $\delta V_{12}$, so that the flavor anomalies match at all points where $v \neq 0$ [1]. At the origin $V^{i j}=0$, the full flavor group is unbroken, and the tangent vectors $\delta V^{i j}$ are unconstrained. The anomalies in the UV and IR theories are computed using the fields in Table $\mathbb{1}$, and match only for the case of $N_{F}=3$ [1].

The point

$$
Q_{0}=w\left(\begin{array}{ccccc}
1 & 0 & 0 & \cdots & 0 \\
0 & 1 & 0 & \cdots & 0
\end{array}\right)
$$

with $w^{2}=v$ projects to a point Eq. (49) on the classical moduli space. As before, $Q_{0}$ is a point on a closed orbit that completely breaks the gauge group, so anomaly matching is guaranteed. This explains all the points where the anomalies match for $N_{F} \neq 3$. In the case where $N_{F}=3$, the classical moduli space is described by a superpotential 


$$
W \propto \operatorname{Pf} V
$$

In this case, Theorem 4 implies that $S U(2) \times S U\left(2 N_{F}-2\right) \times U(1)_{R}$ anomaly matching at $V^{i j} \neq 0$ also implies that the anomalies for this subgroup match at the origin. But that is sufficient to guarantee anomaly matching for the full $S U\left(2 N_{F}\right) \times U(1)_{R}$ flavor symmetry at the origin.

$$
\text { 2. } N_{c}>2
$$

The analysis can be repeated for the case $N_{c}>2$. The computations are more involved than for $N_{c}=2$, because it is tedious to find the tangent vectors at a given point on the moduli space. One can show that all points where anomalies match between the UV and IR theories are covered by the anomaly matching theorem, except for trivial cases in which the unbroken flavor symmetry group is anomaly-free. Instead of going through a detailed description of anomaly matching at the different points of the moduli space, we will illustrate the anomaly matching at one interesting point (the "baryon point") $\hat{\phi}_{0}$ on the moduli space for $N_{F} \geq N_{c}$, where $M_{j}^{i}=0, B^{12 \cdots N_{c}}=1$ (with all other components zero), $\tilde{B}=0$.

The unbroken flavor group at $\hat{\phi}_{0}$ is $S U\left(N_{c}\right)_{L} \times S U\left(N_{F}-N_{c}\right)_{L} \times S U\left(N_{F}\right)_{R} \times U(1)_{B} \times U(1)_{R}$. The point $\phi_{0}$

$$
Q_{0}=\left(\begin{array}{cccc|ccc}
1 & 0 & 0 & 0 & 0 & \cdots & 0 \\
0 & 1 & 0 & 0 & 0 & \cdots & 0 \\
\vdots & & & & & \vdots \\
0 & 0 & 0 & 1 & 0 & \cdots & 0
\end{array}\right), \quad \tilde{Q}_{0}=0
$$

projects to $\hat{\phi}_{0}, \pi\left(\phi_{0}\right)=\hat{\phi}_{0}$. Theorems 2 and 3 tells us that the anomalies should match at this point. The tangent vectors on the classical moduli space at $\hat{\phi}_{0}$ can be found easily in this case, since $\pi_{\phi}^{\prime}$ is onto. They are given by $\delta M_{j}^{i}, i \leq N, \delta B^{1 \ldots N_{c}}$ and $\delta B^{1 \ldots \hat{k} \ldots N_{c} r}$, where $1 \leq k \leq N_{c}, r>N_{c}$, and $\hat{k}$ means that the value $k$ is omitted. The transformation properties of the fundamental and composite fields is given in Table III. The anomalies in the UV and IR theories are tabulated in Table $\mathbb{E}$. Clearly, it is simpler to use the anomaly matching theorem, instead of computing the entries in Table IV.

\section{S-confinement and Quantum Deformations}

For any value of $N_{c}$ and $N_{F}=N_{c}+1$ the classical moduli space of supersymmetric QCD is the set of critical points of the flavor invariant superpotential $M_{j}^{i} B_{i} \tilde{B}^{j}-\operatorname{det} M$. The effective superpotential is therefore [2]

$$
W_{0}=\frac{1}{\Lambda^{2 N_{c}-1}}\left(M_{j}^{i} B_{i} \tilde{B}^{j}-\operatorname{det} M\right)
$$

and the quantum moduli space $\mathcal{M}_{\Lambda}$ agrees with $\mathcal{M}_{\mathrm{cl}}$. The matching of the anomalies of the $S U\left(N_{c}\right)_{L} \times S U\left(N_{F}-N_{c}\right)_{L} \times S U\left(N_{F}\right)_{R} \times U(1)_{B} \times U(1)_{R}$ flavor subgroup unbroken at the "baryon" point of the previous subsection, together with that of the "antibaryon" point 
TABLE III. Flavor transformations of the fundamental and composite fields at a "baryon point." The quark fields are divided up into $Q_{1}^{i \alpha}$ and $Q_{2}^{i \alpha}$, which are $Q^{i \alpha}, i \leq N_{c}$ and $Q^{i \alpha} i>N_{c}$, respectively. The tangent vectors on the moduli space are $\delta M_{j}^{i}, i \leq N_{c}, \delta B^{1 \ldots N}$ and $\delta B^{1 \ldots \hat{k} \ldots N_{c} r}, r>N_{c}, k \leq N_{c}$.

\begin{tabular}{cccccc}
\hline \hline & $S U\left(N_{c}\right)_{L}$ & $S U\left(N_{F}-N_{c}\right)_{L}$ & $S U\left(N_{F}\right)_{R}$ & $B$ & $R$ \\
\hline$Q_{1}^{i \alpha}$ & $\square$ & - & - & 0 & 0 \\
$Q_{2}^{i \alpha}$ & - & $\square$ & - & $-N_{F}$ & $\left(2 N_{F}-2 N_{c}\right) /\left(2 N_{F}-N_{c}\right)$ \\
$\tilde{Q}_{j \alpha}$ & - & - & $\square$ & $N_{F}-N_{c}$ & $\left(2 N_{F}-2 N_{c}\right) /\left(2 N_{F}-N_{c}\right)$ \\
\hline$\delta M_{j}^{i}$ & $\square$ & - & $\square$ & $N_{F}-N_{c}$ & $\left(2 N_{F}-2 N_{c}\right) /\left(2 N_{F}-N_{c}\right)$ \\
$\delta B^{1 \ldots N}$ & - & - & - & 0 & 0 \\
$\delta B^{1 \ldots \hat{k} \ldots N_{c} r}$ & $\square$ & $\square$ & - & $-N_{F}$ & $\left(2 N_{F}-2 N_{c}\right) /\left(2 N_{F}-N_{c}\right)$ \\
\hline \hline
\end{tabular}

TABLE IV. Flavor anomalies in the UV and IR theories due to the various fields. $\lambda$ is the gaugino. Here $s=N_{F}-N_{c}, t=-N_{c} /\left(2 N_{F}-N_{c}\right)$. See the Table III caption for the definition of the various fields.

\begin{tabular}{c|cccc|ccc}
\hline \hline & \multicolumn{3}{|c|}{$\mathrm{UV}$} & \multicolumn{3}{c}{$\mathrm{IR}$} \\
\hline & $Q_{1}^{i \alpha}$ & $Q_{2}^{i \alpha}$ & $\tilde{Q}_{j \alpha}$ & $\lambda$ & $\delta M_{j}^{i}$ & $\delta B^{1 \ldots N}$ & $\delta B^{1 \ldots k \ldots N_{c} r}$ \\
\hline$S U\left(N_{c}\right)_{L}^{3}$ & $N_{c}$ & 0 & 0 & 0 & $N_{F}$ & 0 & $-s$ \\
$S U(s)_{L}^{3}$ & 0 & $N_{c}$ & 0 & 0 & 0 & 0 & $N_{c}$ \\
$S U\left(N_{F}\right)_{R}^{3}$ & 0 & 0 & $-N_{c}$ & 0 & $-N_{c}$ & 0 & 0 \\
$B^{3}$ & 0 & $-N_{c} s N_{F}^{3}$ & $N_{c} N_{F} s^{3}$ & 0 & $N_{c} N_{F} s^{3}$ & 0 & $-N_{c} s N_{F}^{3}$ \\
$R^{3}$ & $-N_{c}^{2}$ & $N_{c} s t^{3}$ & $N_{c} N_{F} t^{3}$ & $N_{c}^{2}-1$ & $N_{c} N_{F} t^{3}$ & -1 & $N_{c} s t^{3}$ \\
$S U\left(N_{c}\right)_{L}^{2} B$ & 0 & 0 & 0 & 0 & $N_{F} s$ & 0 & $-s N_{F}$ \\
$S U(s)_{L}^{2} B$ & 0 & $-N_{c} N_{F}$ & 0 & 0 & 0 & 0 & $-N_{c} N_{F}$ \\
$S U\left(N_{F}\right)_{R}^{2} B$ & 0 & 0 & $N_{c} s$ & 0 & $N_{c} s$ & 0 & 0 \\
$S U\left(N_{c}\right)_{L}^{2} R$ & $-N_{c}$ & 0 & 0 & 0 & $N_{F} t$ & 0 & $s t$ \\
$S U(s)_{L}^{2} R$ & 0 & $N_{c} t$ & 0 & 0 & 0 & 0 & $N_{c} t$ \\
$S U\left(N_{F}\right)_{R}^{3} R$ & 0 & 0 & $N_{c} t$ & 0 & $N_{c} t$ & 0 & 0 \\
$B^{2} R$ & 0 & $N_{c} s N_{F}^{2} t$ & $N_{c} N_{F} s^{2} t$ & 0 & $N_{c} N_{F} s^{2} t$ & 0 & $N_{c} s N_{F}^{2} t$ \\
$R^{2} B$ & 0 & $-N_{c} N_{F} s t^{2}$ & $N_{c} N_{F} s t^{2}$ & 0 & $N_{c} N_{F} s t^{2}$ & 0 & $-N_{c} s N_{F} t^{2}$ \\
$B$ & 0 & $-N_{c} s N_{F}$ & $N_{c} N_{F} s$ & 0 & $N_{c} N_{F} s$ & 0 & $-N_{c} s N_{F}$ \\
$R$ & $-N_{c}^{2}$ & $N_{c} s t$ & $N_{c} N_{F} t$ & $N_{c}^{2}-1$ & $N_{c} N_{F} t$ & -1 & $N_{c} s t$ \\
\hline \hline
\end{tabular}


(with $Q \leftrightarrow \tilde{Q}$ ) with unbroken flavor subgroup $S U\left(N_{c}\right)_{R} \times S U\left(N_{F}-N_{c}\right)_{R} \times S U\left(N_{F}\right)_{L} \times$ $U(1)_{B} \times U(1)_{R}$ imply the matching for the full flavor group at the origin (Theorem 4). Another application of Theorem 4 proves 't Hooft's conditions are satisfied at any vacuum in the moduli space. According to the discussion of Section [V, adding a tree level mass term $m Q^{N_{c}+1 \alpha} \tilde{Q}_{\alpha N_{c}+1}$ to $W$ gives the effective superpotential

$$
W_{m}=\frac{1}{\Lambda^{2 N_{c}-1}}\left(M_{j}^{i} B_{i} \tilde{B}^{j}-\operatorname{det} M\right)+m M_{N_{c}+1}^{N_{c}+1} .
$$

The critical points of Eq. (55) give the quantum moduli space

$$
\begin{aligned}
M_{N_{c}+1}^{i} & =M_{j}^{N_{c}+1}=0, \\
B_{i} & =\tilde{B}^{i}=0, i \leq N_{c}, \\
\operatorname{det} M-B \tilde{B} & =\Lambda_{N}^{2 N+1},
\end{aligned}
$$

of the $N_{F}=N_{c}$ theory, where $B=B_{N_{c}+1}, \tilde{B}=\tilde{B}^{N_{c}+1}$ and the determinant extends to the light flavors $i, j \leq N_{c}$. Applying Theorem 4 to $W_{0}$ and $W_{m}$ proves that anomaly matching for the $N_{F}=N_{c}+1$ theory implies anomaly matching at every point of the quantum deformed moduli space of the $N_{F}=N_{c}$ theory. We can therefore avoid the explicit checks done at isolated points of $\mathcal{M}_{\Lambda}$ in [1] and be sure the result holds at every point. Note that, although $\mathcal{M}_{\Lambda}$ of the $N_{F}=N_{c}$ theory can be described as the set of critical points of a superpotential when embedded in the vector space of gauge invariants of the $N_{F}=N_{c}+1$ theory, $\mathcal{M}_{\mathrm{cl}}$ cannot. In particular, anomalies do not match at the point $M_{j}^{i}=0, B=\tilde{B}=0$, which belongs to $\mathcal{M}_{\mathrm{cl}}$ but not to $\mathcal{M}_{\Lambda}$.

\section{B. Other S-confining Theories}

Our analysis for QCD extends to other s-confining theories, an exhaustive list of which can be found in [6]. For all s-confining theories $\mathcal{M}_{\mathrm{cl}}=\mathcal{M}_{\Lambda}$ can be described by a flavor invariant superpotential, so a finite number of points $\phi_{i} \in U$ satisfying the hypothesis of the matching theorem is enough to prove anomaly matching at every point of their moduli space and at the quantum deformed moduli space of the theories obtained from them by integrating out a heavy fields. Explicit verification of this matching both for the s-confining theory and the quantum deformed one is a formidable task. Even the determination of a complete set of basic gauge invariants is sometimes nontrivial. We give one simple example of an s-confining theory.

\section{1. $S P(2 N, \mathbb{C})$ with $2 N+4$ fundamentals $Q^{\alpha i}$}

$S P(2 N, \mathbb{C})$ is the group that leaves invariant the 2 -form

$$
J_{\alpha \beta}=\left(\begin{array}{cc}
0 & \mathcal{I}_{N \times N} \\
-\mathcal{I}_{N \times N} & 0
\end{array}\right) .
$$

This is the complexification of the group $G_{r}=S P(2 N)=S P(2 N, \mathbb{C}) \cap U(2 N)$. Just one point $\phi_{0}$ is required to prove anomaly matching in the s-confining $S P$ theory with $2 N+4$ 
fundamentals $Q^{\alpha i}, Q_{0}^{\alpha i}=m \delta^{\alpha i}, i \leq 2 N, 0$ for $i>2 N$. This point totally breaks $G$, and its orbit is the closed set $S P(2 N, \mathbb{C})$, naturally embedded in the vector space $\mathbb{C}^{2 N(2 N+4)}$ of $2 N \times(2 n+4)$ matrices.

\section{CONCLUSIONS}

For supersymmetric gauge theories with a reductive gauge group $G$, the classical moduli space $\mathcal{M}_{\mathrm{cl}}$ is the algebraic quotient of the algebraic set of critical points of the superpotential under the action of $G$. There are known bounds for its dimension, which can be determined without knowing the basic invariant polynomials when closed orbits of maximum dimension are found. The anomaly matching theorem can be used to show that the 't Hooft consistency conditions are satisfied at points that totally break the complexification of the gauge group and either have a closed orbit or are mapped onto a smooth point of $\mathcal{M}_{\mathrm{cl}}$. Anomalies will match at every point of $\mathcal{M}_{\Lambda}$ if a few suitable points satisfying the above hypothesis are found and both $\mathcal{M}_{\mathrm{cl}}$ and $\mathcal{M}_{\Lambda}$ can be described as the set of critical points of a flavor invariant superpotential. Anomaly matching for a theory implies anomaly matching for those theories obtained from it by integrating out matter, even when these have a quantum modified moduli space. Anomalies match for the large family of s-confining theories and those obtained from them by integrating out a flavor, which have a quantum modified moduli space. The explicit anomaly computations found in the literature can often be avoided, if one uses the results discussed here. These results also allow one to anticipate if $\mathcal{M}_{\mathrm{cl}}$ or a quantum deformed $\mathcal{M}_{\Lambda}$ describes correctly the IR massless modes, or if an alternative description (such us a dual theory) is required. Extensions of our results to cases where the complexification of the gauge group is not totally broken and applications to dual theories is currently under study.

\section{ACKNOWLEDGMENTS}

We are indebted to M.L. Barberis, M. Hunziker and N. Wallach,for extensive discussions on algebraic geometry, and to $\mathrm{N}$. Wallach for giving us a copy of his book [9] prior to publication. We are also grateful to G. Schwarz for telling us about Knop's theorem, and for discussion about when $\pi^{\prime}$ can be surjective. This work was supported in part by a Department of Energy grant DOE-FG03-97ER40546. 


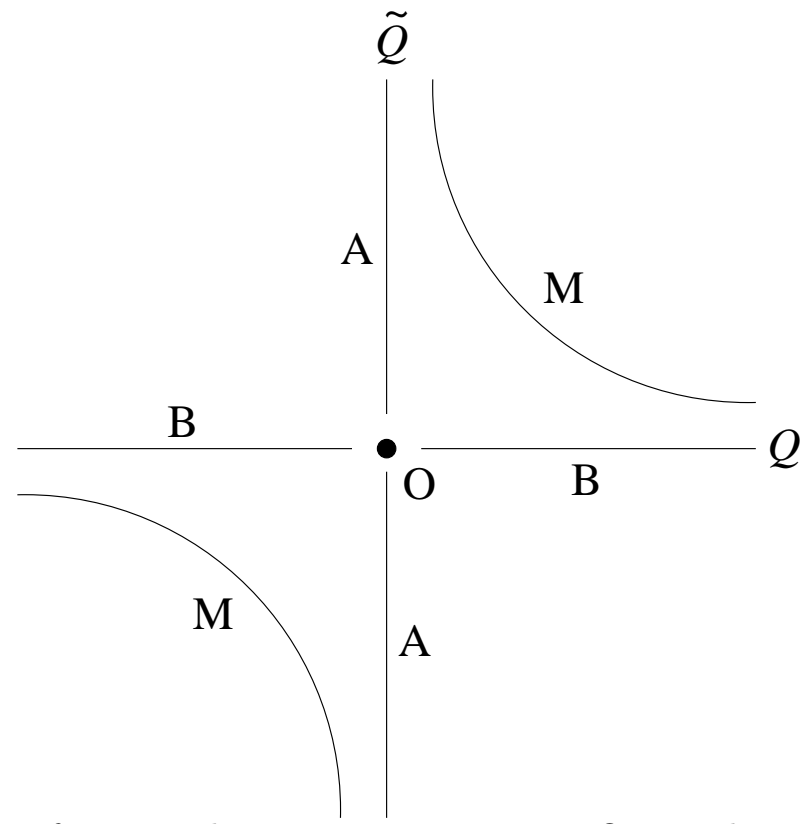

FIG. 1. The structure of gauge orbits in supersymmetric QED. The orbits are connected. They appear disconnected because the figure only shows the restriction of the configuration space to real values of $Q$ and $\tilde{Q}$. The orbits $M$ and $O$ are closed, and $A$ and $B$ are not closed. $M$, and $A \cup B \cup O$ are fibers.

\section{REFERENCES}

[1] N. Seiberg, Phys. Rev. D49 (1994) 6857, Nucl. Phys. B435 (1995), 129.

[2] K. Intriligator, R.G. Leigh, N. Seiberg, Phys. Rev. D50 (1994) 1092, K. Intriligator and N. Seiberg, Nucl. Phys. B444 (1995) 125. For a more detailed list of references, see K. Intriligator and N. Seiberg hep-th/9509066 and M.E. Peskin hep-th/9702014.

[3] G. 't Hooft, Naturalness, Chiral Symmetry and Chiral Symmetry Breaking, Lectures given at the Cargèse Summer Institute, Cargèse, France, 1979.

[4] G. Dotti and A.V. Manohar, UCSD/PTH 97-13, hep-th/9706075.

[5] M.A. Luty and W. Taylor IV, Phys. Rev. D53 (1996) 3399.

[6] C. Csaki, M. Schmaltz and W. Skiba, Phys. Rev. D55 (1997) 7840.

[7] J. Wess and J. Bagger, Supersymmetry and Supergravity, Second Edition, (Princeton University Press, Princeton, 1992).

[8] I.R. Shafarevich, Basic Algebraic Geometry, Vol. I, Second Edition, (Springer-Verlag, Berlin, 1994).

[9] R. Goodman and N.R. Wallach, Representation and Invariants of the Classical Groups, (Cambridge University Press, Cambridge, 1997).

[10] V.L. Popov and E.B. Vinberg, Invariant Theory, in Algebraic Geometry IV: Linear Algebraic Groups, Invariant Theory, ed. A.N. Parshin and I.R. Shafarevich, (SpringerVerlag, Berlin, 1994).

[11] F. Knop, Manuscripta Math. 56 (1986) 419.

[12] D. Luna, Bull. Math. Soc. Fr., Suppl. Mém 33 (1973) 81 (Quoted in [10], Theorem 6.1). 


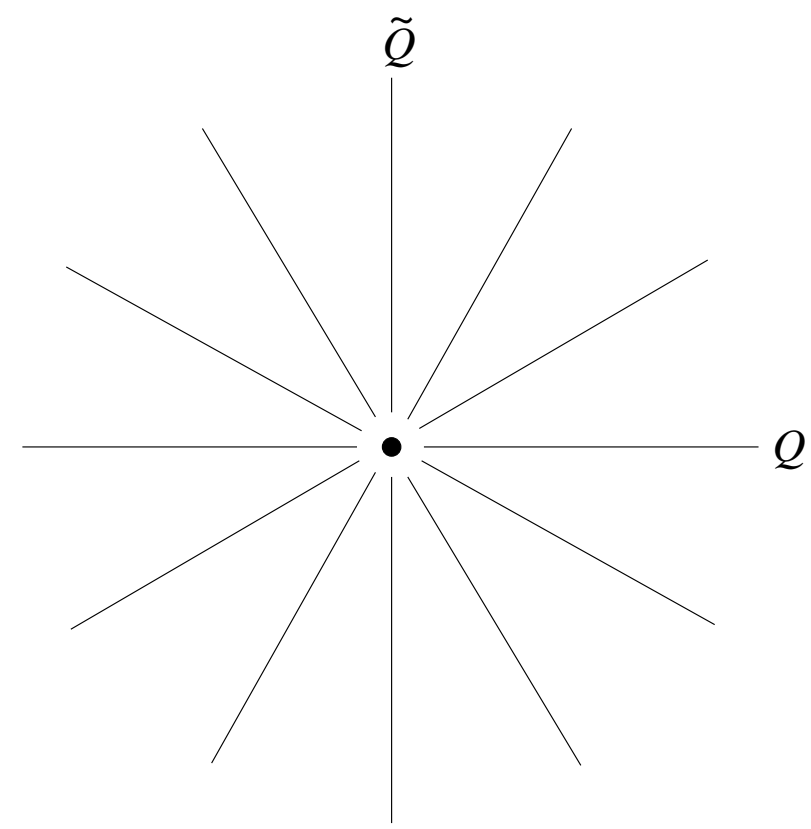

FIG. 2. The structure of gauge orbits in supersymmetric QED with equal charges. Only the projection onto real values of $Q_{1}$ and $Q_{2}$ is shown. The orbits of a generic point $\left(Q_{1}, Q_{2}\right)$ are radial lines, and are not closed. The orbit of $(0,0)$ is a single point, and is closed. There is a single fiber which is $\mathbb{C}^{2}$.

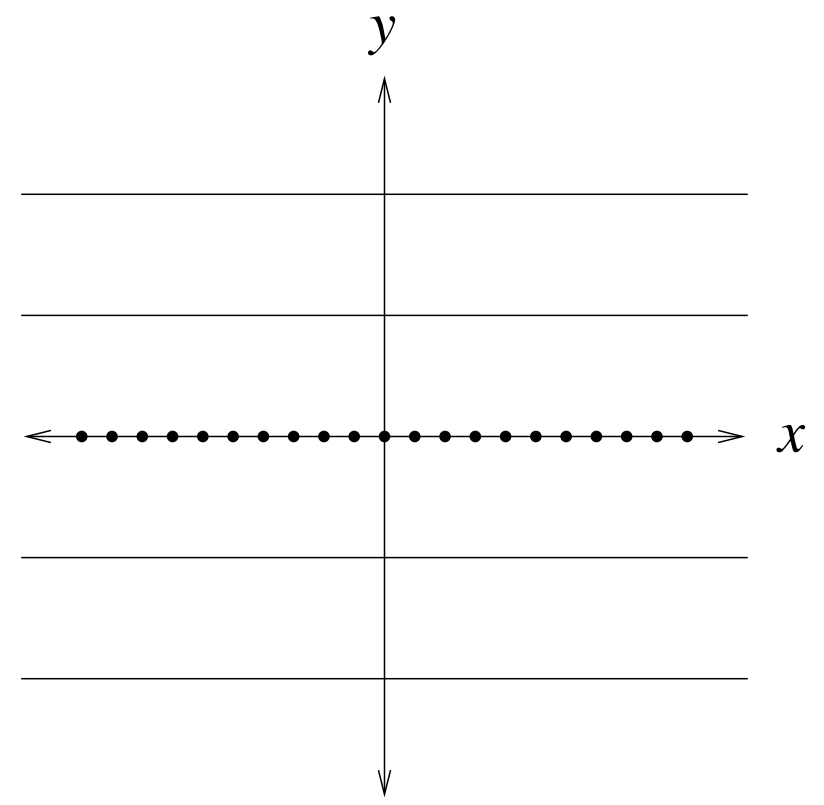

FIG. 3. The structure of gauge orbits for the non-reductive group $\mathbb{C}^{+}$. The orbits are horizontal lines if $y \neq 0$, and points if $y=0$. The fibers are the horizontal lines $y=$ constant. The fiber $y=0$ contains an infinite number of closed orbits. 